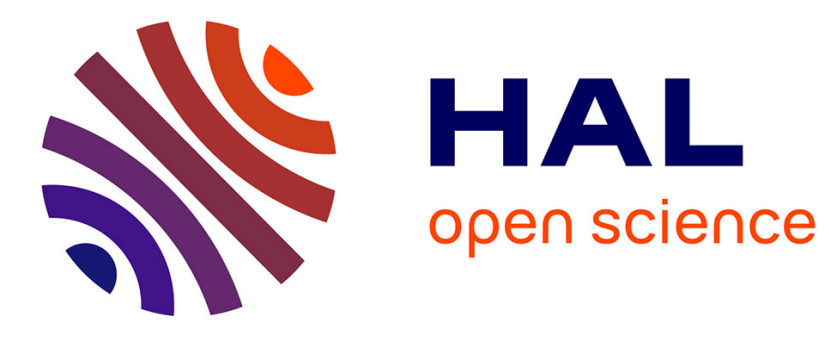

\title{
Precise Extraction of Charge Carrier Mobility for Organic Transistors
}

\author{
Yong Xu, Yun Li, Songlin Li, Francis Balestra, Gérard Ghibaudo, Wenwu Li, \\ Yen-Fu Lin, Huabin Sun, Jing Wan, Xinran Wang, et al.
}

\section{- To cite this version:}

Yong $\mathrm{Xu}$, Yun Li, Songlin Li, Francis Balestra, Gérard Ghibaudo, et al.. Precise Extraction of Charge Carrier Mobility for Organic Transistors. Advanced Functional Materials, 2020, Emerging Thin-Film Transistor Technologies and Applications, 30 (20), pp.1904508. 10.1002/adfm.201904508 . hal-02998279

\section{HAL Id: hal-02998279 \\ https://hal.science/hal-02998279}

Submitted on 23 Nov 2020

HAL is a multi-disciplinary open access archive for the deposit and dissemination of scientific research documents, whether they are published or not. The documents may come from teaching and research institutions in France or abroad, or from public or private research centers.
L'archive ouverte pluridisciplinaire HAL, est destinée au dépôt et à la diffusion de documents scientifiques de niveau recherche, publiés ou non, émanant des établissements d'enseignement et de recherche français ou étrangers, des laboratoires publics ou privés. 


\title{
WILEY-VCH
}

DOI: 10.1002/adma.((please add manuscript number))

Article type: Review

\section{Precise Extraction of Charge Carrier Mobility for Organic Transistors}

Yong Xu*, Yun Li, Songlin Li, Francis Balestra, Gerard Ghibaudo, Wenwu Li, Yen-Fu Lin, Huabin Sun*, Jing Wan*, Xinran Wang, Yufeng Guo*, Yi Shi, and Yong-Young Noh*

Prof. Y. Xu, Prof. H.B. Sun, Prof. J. Wan, Prof. Y.F. Guo

School of Electronic and Optical Engineering \& College of Microelectronics, Nanjing University of Posts and Telecommunications, Nanjing, 210023, China

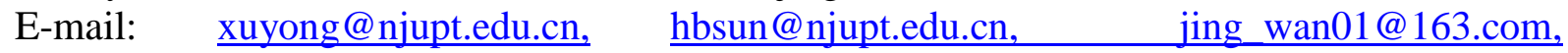
yfguo@njupt.edu.cn

Prof. Y. Li, Prof. S.L. Li, Prof. X.R. Wang, Prof. Y. Shi

School of Electronic Science and Engineering, Collaborative Center of Advanced Microstructures, National Laboratory of Solid-State Microstructures, Nanjing University, Nanjing 210093, China

Prof. F. Balestra, Prof. G. Ghibaudo

IMEP-LAHC, Grenoble-INP, MINATEC,

3 Parvis Louis Neel, BP 257, 38016 Grenoble, France

Prof. W.W. Li

Key Laboratory of Polar Materials and Devices, Ministry of Education, Department of Electronic Engineering, East China Normal University, Shanghai 200241, China

Prof. Y.F. Lin

Department of Physics, National Chung Hsing University, Taichung 40227, Taiwan

Prof. Y.Y. Noh

Department of Chemical Engineering, Pohang University of Science and Technology (POSTECH), 77 Cheongam-Ro, Nam-Gu, Pohang 37673, Republic of Korea

E-mail: yynoh@postech.ac.kr

\begin{abstract}
Unreliable mobility values, and particularly greatly overestimated values and severely distorted temperature dependences, have recently hampered the development of the organic transistor field. Given that organic field-effect transistors (OFETs) have been routinely used to evaluate the mobility, precise parameter extraction using the electrical properties of OFETs is thus of primary importance. This review examines the origins of the various mobilities that must be determined for OFET applications, the relevant extraction methods, and the data selection limitations, which help in avoiding conceptual errors during mobility extraction. For increased precision, the review also discusses device fabrication considerations, calibration of both the specific gate-dielectric capacitance and the threshold voltage, the contact effects, and the bias and temperature dependences, which must actually be handled with great care but have mostly been overlooked to date. This paper serves as a systematic overview of the OFET mobility extraction process to ensure high precision and will also aid in improving future research.
\end{abstract}

Keywords: Mobility, organic transistor, parameter extraction, reliability 


\section{WILEY-VCH}

\section{Introduction}

Mobility assessment has become a critical issue for organic transistors. ${ }^{[1]}$ Pioneering researchers consistently suggested conservative methods to report mobility values based on the use of organic field-effect transistors (OFETs), ${ }^{[2-4]}$ similar to the setup shown in Figure 1a. This method was used in the context that OFETs have been routinely used in approaches to evaluate mobility, but many of these devices are affected by extrinsic effects that could cause misinterpretation of the measured mobility values and the related temperature dependence, and may possibly have misled development in this field. For example, high contact resistance can control the OFET switching process, leading to late channel accumulation (i.e., a high threshold voltage) and then a steep turn-on characteristic. ${ }^{[5,6]}$ The latter can be translated into high mobility as an artefact because the mobility is extracted by linear fitting of a narrow range of the data that corresponds to the steeply increasing drain current. An illogical phenomenon may then appear where the corresponding OFETs with ohmic contacts deliver higher drain currents but indicate lower mobilities (Figure 1b); this occurs because the wellfunctioning OFETs provide greater linearity during mobility extraction and thus a constant and more intrinsic but seemingly "low" mobility is obtained over a wide range of the device data. ${ }^{[4,7]}$ Obviously, higher output currents for OFETs with identical device configurations (i.e., specific gate dielectric capacitance, and channel width and length) should point to higher mobility. This example illustrates that both device optimization and the parameter extraction process are important for mobility assessment. Rather than exhaustive device optimization that is uncertain for unknown materials or technologies, this paper focuses on mobility extraction, which is a matter that requires attention every day.

Recently, several papers have been published on mobility extraction, ${ }^{[4,8,9]}$ the roots of the misconceptions about mobility and the relevant extraction methods; however, a systematic illustration of the problem is still lacking. Additionally, determination of ways to avoid errors in mobility evaluation, including device fabrication aspects, the measurement setup configuration, and the bias and temperature dependences, remains an urgent problem. For example, the most extensively used field-effect mobility is not actually derived from the transconductance; in fact, it is mainly calculated from the square root of the saturation current, which means that it is actually the saturation mobility. Additionally, the linear fitting for mobility extraction is performed at low gate voltages, particularly around the threshold voltage, in an attempt to gain the highest possible mobility, ${ }^{[5,6]}$ as shown in Figure 1c. Apart from the causes documented in the literature, such as the contact resistance, ${ }^{[10-12]}$ this type of extraction may be conceptually inappropriate because the related method presumes a charge approximation that is only valid in the strong accumulation regime, i.e., at high gate voltages. ${ }^{[13]}$ Alternatively, ambipolar conduction may predominate the channel conduction at low gate voltages during measurement of the transfer characteristics in the saturation regime; see Figure $1 \mathrm{~d} .{ }^{[14]}$ The extracted mobility can also be confused by the coexistence of holes and electrons within the channel. $^{[8,15,16]}$

Taking this situation into consideration, this review therefore commences with definitions of the mobilities that are commonly used for OFET applications to explain their underlying physical meanings explicitly. After that, the associated extraction methods are revisited and the significant factors that affect the mobility reliability are discussed. Finally, the review addresses the essential effects that must be considered when performing mobility extraction, including device preparation, specific gate-dielectric capacitance calibration, threshold voltage calibration, contact effects, bias dependences, and temperature dependence.

\section{Mobilities}

The various mobilities of OFETs can be classified simply into two categories: the bulk mobility and the field-effect transistor (FET) mobility. The former category is for bulk semiconductors with a homogeneous charge distribution, where lattice vibrations (phonon scattering), 


\section{WILEY-VCH}

energetic disorders, and perhaps ionized impurity scattering (affecting the doping density) can degrade the mobility. The FET mobility occurs in transistors in which the high-density charges are distributed near the dielectric-semiconductor interface. The FET mobility thus suffers more from extrinsic effects, e.g., from surface roughness scattering, remote Coulombic scattering, and the contact resistance. Each of these limiting factors corresponds to one mobility type. The overall apparent mobility is based on the lowest value, as described by Matthiessen's rule: ${ }^{[17]}$

$$
\frac{1}{\mu}=\frac{1}{\mu_{1}}+\frac{1}{\mu_{2}}+\frac{1}{\mu_{3}}+\cdots
$$

Despite the diversity of mobility types, this review concentrates on the few mobilities that are used regularly in OFETs only. Other mobilities like those extracted by using space-charge limited current (SCLC), ${ }^{[18-20]}$ time of flight (TOF),${ }^{[21-24]}$ and charge extraction by linearly increasing voltage (CELIV) may be superior in terms of reliability, ${ }^{[25]}$ while they are not popularly utilized for OFETs. Interested readers can refer to the literature for more details.

(a)
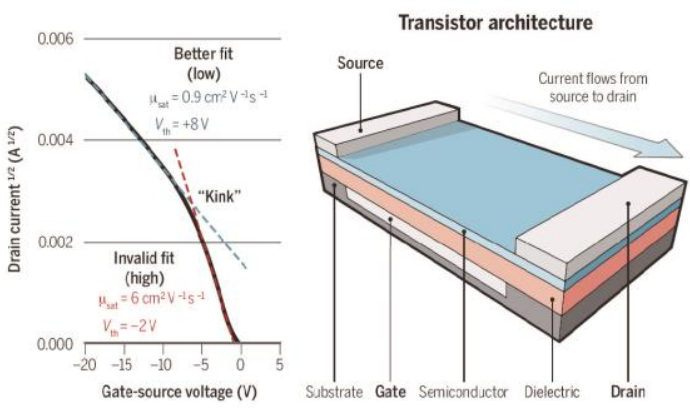

(b)
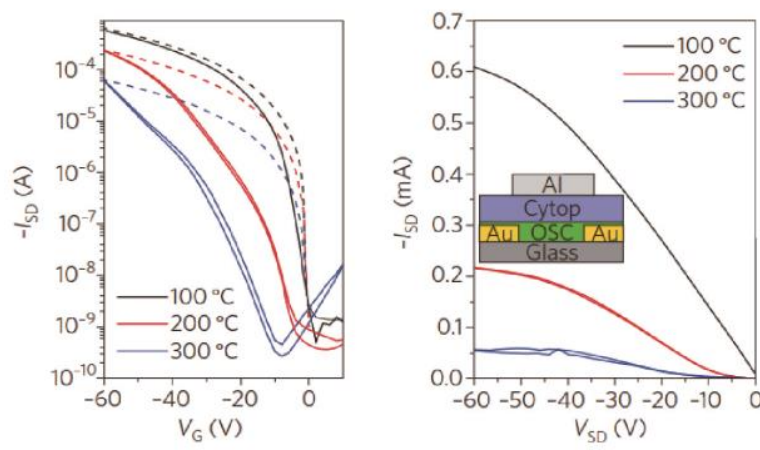

(c)
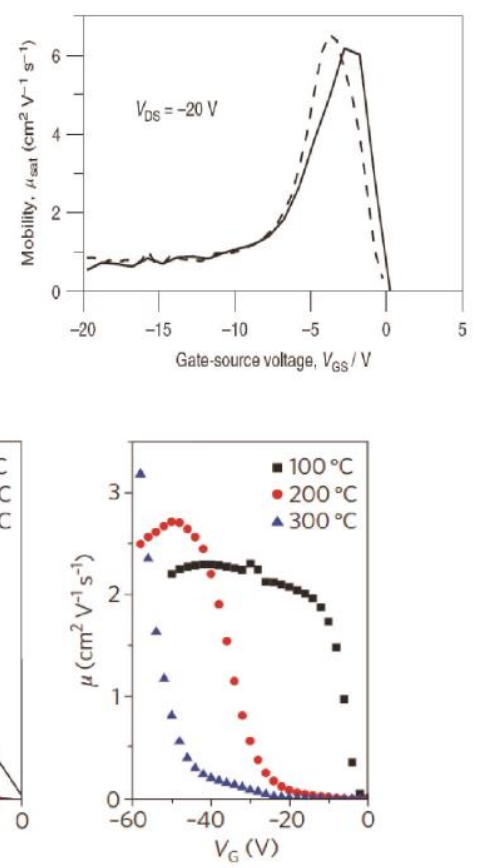

(d)
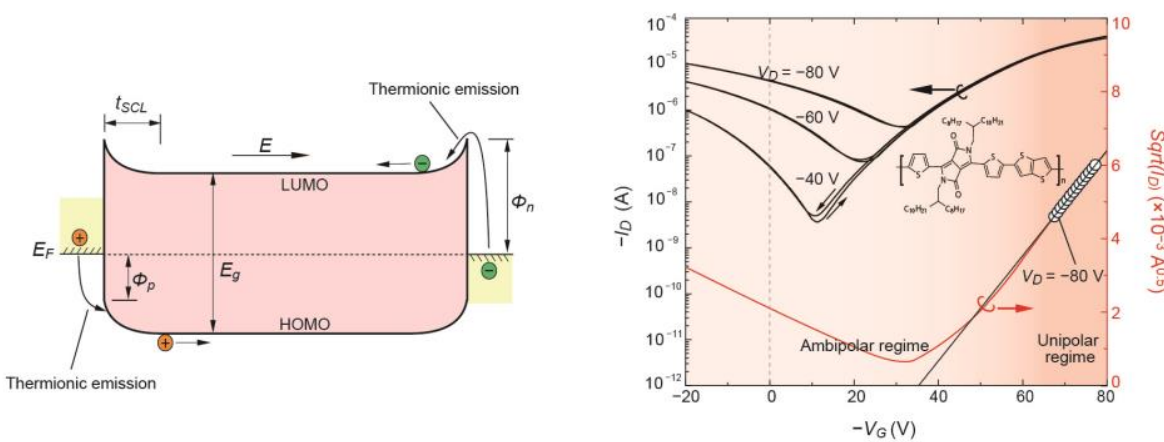

Figure 1. Context for the OFET mobility extraction process. (a) A conservative method was originally proposed to report the mobility when using OFETs. Left: The fitting data slope is first high (giving high mobility) and then changes to low (giving low mobility), while a kink appears between these stages. The high mobility may be a result of the rapidly reduced contact resistance. Right: A typical OFET structure. ${ }^{[3]}$ Copyright 2016, AAAS. (b) Illustration of mobility extraction with nonidealities. A nonideal indacenodithiophene-co-benzothiadiazole (IDTBT) OFET annealed at $100^{\circ} \mathrm{C}$ delivers lower current when compared with a near-ideal device annealed at $300^{\circ} \mathrm{C}$, while the extracted mobilities show an illogical contrast. ${ }^{[4]}$ Copyright 2018, Macmillan. (c) Mobility extracted as a function of gate voltage. A peak is often observed around the threshold voltage itself because of a significant contact effect. $^{[6]}$ Copyright 2018, Macmillan. (d) Illustration of ambipolar conduction. Left: Band diagram of OFET with ambipolar conduction. Right: Typical transfer curves showing ambipolar characteristics. The mobili- 
ty extraction process is confused by the co-existence of holes and electrons within the channel. ${ }^{[14]}$ Copyright 2016, Wiley.

\subsection{Effective Mobility}

The effective mobility is the averaged mobility of all charge carriers involved in the channel. For a transistor with channel width $W$ and length $L$, the drain current $I_{D}$ comprises drift and diffusion components and can be expressed as

$$
I_{D}=\frac{W}{L} \int_{0}^{V_{D}} \mu_{e f f} Q_{i} d U_{c}
$$

where $\mu_{e f f}$ is the effective mobility, $Q_{i}$ is the mobile charge density per unit channel area, $V_{D}$ is the drain voltage, and $U_{C}$ is the quasi-Fermi level. Application of a very small $V_{D}$ maintains a uniform charge distribution along the channel so that the diffusion current can be suppressed. $I_{D}$ can then be simplified to be given by $(W / L) \mu_{\text {eff }} Q_{i} V_{D}$ and $\mu_{\text {eff }}$ is then extracted using ${ }^{[26]}$

$$
\mu_{e f f}=\frac{L}{W} \frac{g_{d}}{Q_{i}}
$$

where $g_{d}$ is the output conductance, which is defined as

$$
g_{d}=\left.\frac{\partial I_{D}}{\partial V_{D}}\right|_{V_{G}=\text { constant }}
$$

where $V_{G}$ is the gate voltage. This case with a variable $V_{D}$ and a constant $V_{G}$ corresponds to measurement of the output characteristics, but in the linear region only (at small $V_{D}$ ).

To extract $\mu_{e f f}$, it is necessary to determine $Q_{i}$ first. There are two ways to obtain $Q_{i}$. The first is to approximate $Q_{i}$ using

$$
Q_{i}=C_{i}\left(V_{G}-V_{T}\right)
$$

where $C_{i}$ is the specific gate-dielectric capacitance per unit area and $V_{T}$ is the threshold voltage. This approximation is valid when $V_{G} \gg V_{T}$, i.e., in the strong accumulation regime, as shown in Figure 2b. This high- $V_{G}$ regime also minimizes the diffusion current, which is a dominant contributor in the sub-threshold regime. From another viewpoint, this approximation offers a different way to extract $\mu_{\text {eff }}$ using the linear transfer characteristics:

$$
I_{\text {Dlin }}=\frac{W}{L} \mu_{e f f} C_{i}\left(V_{G}-V_{T}\right) V_{D}
$$

where $V_{G}$ is variable and $V_{D}$ has a small constant value. The extraction methods will be described in more detail below.
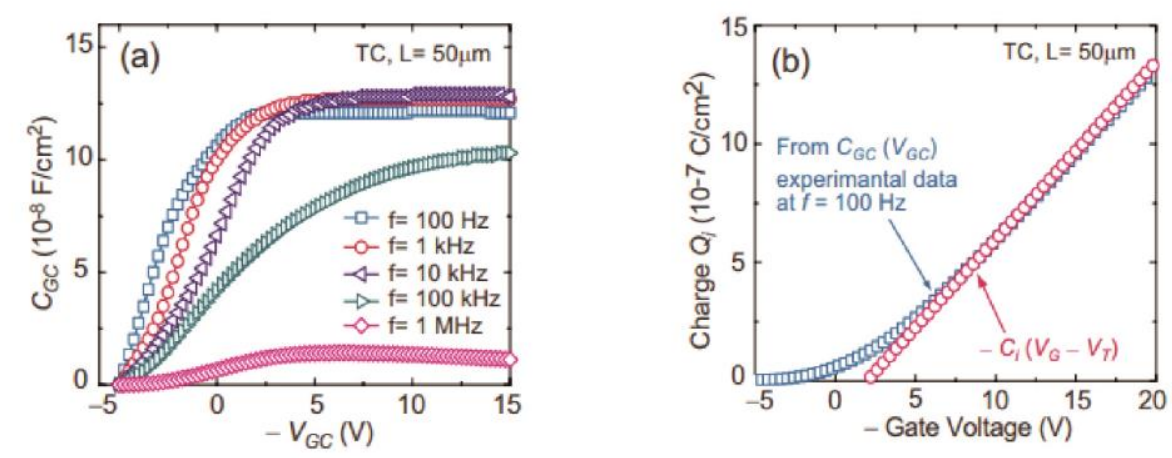

Figure 2. Measurement of charge density using the capacitance-voltage $(C-V)$ characteristics. (a) $C$ - $V$ characteristics of a top-contact, bottom-gate pentacene OFET at various frequencies. (b) Charge densities measured using the $C-V$ characteristics and approximated using $Q_{i}=C_{i}\left(V_{G}-V_{T}\right)$. These two densities coincide at higher gate voltages. ${ }^{[27]}$ Copyright 2010, AIP.

In the approximation above, $C_{i}$ and $V_{T}$ must be determined precisely. However, $C_{i}$ is usually calculated using a nominal dielectric constant and thickness and thus may differ from the actual value to a notable extent. In addition, $V_{T}$ loses its original physical meaning, as in silicon metal-oxide-semiconductor field-effect transistors (MOSFETs), and can be influenced by a variety of uncontrollable effects in OFETs, ${ }^{[28-30]}$ which means that $V_{T}$ is not explicit. These factors combine to make the $\mu_{\text {eff }}$ extraction ambiguous. In fact, $Q_{i}$ can be measured directly 


\section{WILEY-VCH}

using the capacitance-voltage $(C-V)$ characteristics, as shown in Figure 2a. This is the second way to obtain $Q_{i}$, which is given by

$$
Q_{i}=\int_{-\infty}^{V_{G}} C_{G C} d V_{G}
$$

where $C_{G C}$ is the gate-to-channel capacitance. This experimentally measured $Q_{i}$ avoids the prerequisite of knowing the values of $C_{i}$ and $V_{T}$. Therefore, the capacitance-based $Q_{i}$ method is much simpler and more accurate when compared with the charge approximation-based method to extract $\mu_{\text {eff. }}{ }^{[27,31,32]}$

\subsection{Low-Field Mobility}

Because a higher $V_{G}$ applies in this case, charges are attracted towards the dielectricsemiconductor interface and the surface influence thus becomes stronger. $\mu_{\text {eff }}$ declines with

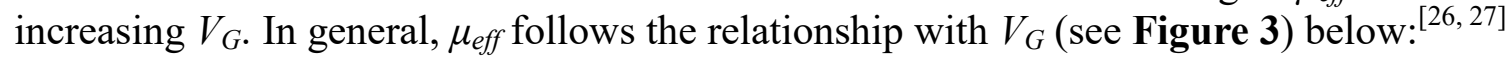

$$
\mu_{e f f}=\frac{\mu_{0}}{1+\theta\left(V_{G}-V_{T}\right)}
$$

where $\mu_{0}$ is the low-field mobility and $\theta$ is the mobility attenuation factor.

In conventional Si MOSFETs, $\theta$ contains several contributions stemming from surface roughness scattering, quantization effects and other effects, and thus has positive values in

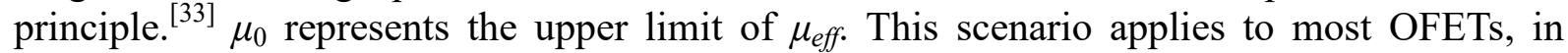
which scattering caused by dielectric dipoles and the limitations of the contact resistance may play important roles. ${ }^{[27]}$ However, in some OFETs with very disordered organic semiconductor (OSC) or without appropriate optimization, $\mu_{\text {eff }}$ can be enhanced by increasing $V_{G},{ }^{[34,35]}$ where $\theta$ has negative values. $\mu_{0}$ then represents the lower limit of $\mu_{\text {eff. }}$ Regardless of the value of $\theta$, it can be seen from Equation (8) and Figure 3 that $\mu_{0}$ is $\mu_{\text {eff }}$ when $V_{G}=V_{T}$, i.e., at a low gate field when the channel begins to be established. This implies that $\mu_{0}$ is more like a bulk mobility rather than a surface mobility, so it is more intrinsic when compared with $\mu_{e f f}$.

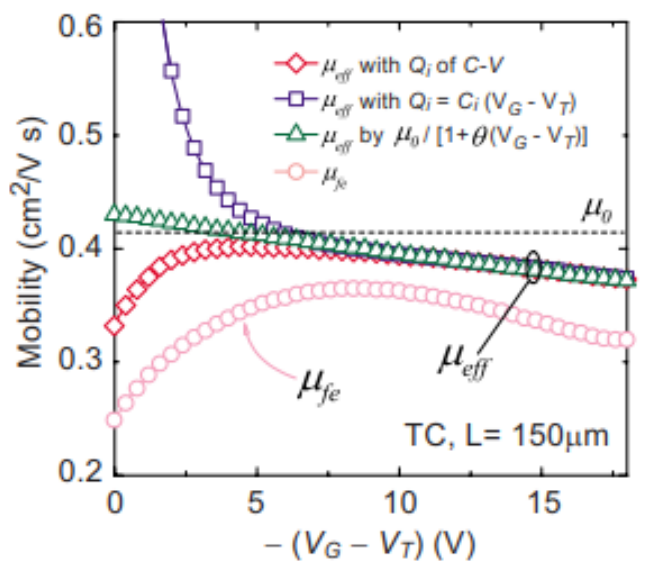

Figure 3. Comparison of the three mobilities extracted from a top-contact, bottom-gate pentacene OFET. ${ }^{[27]}$ Copyright 2010, AIP.

\subsection{Field-Effect Mobility}

The field-effect mobility is derived from the transconductance $g_{m}$, which is defined as

$$
g_{m}=\left.\frac{\partial I_{D}}{\partial V_{G}}\right|_{V_{D}=\text { constant }}
$$

For a transistor operating in the linear regime (under application of a small $V_{D}$ ), and by combining Equation (5) with Equation (8), the drift current in Equation (2) can be rewritten as

Then, $g_{m}$ reads as

$$
I_{\text {Dlin }}=\frac{W}{L} \frac{\mu_{0}}{1+\theta\left(V_{G}-V_{T}\right)} C_{i}\left(V_{G}-V_{T}\right) V_{D}
$$

$$
g_{m}=\left.\frac{\partial I_{D}}{\partial V_{G}}\right|_{V_{D}=\text { constant }}=\frac{W}{L} \frac{\mu_{0}}{\left[1+\theta\left(V_{G}-V_{T}\right)\right]^{2}} C_{i} V_{D}
$$

The field-effect mobility $\mu_{f e}$ is then defined as 


$$
\mu_{f e}=\frac{L}{W} \frac{g_{m}}{C_{i} V_{D}}=\frac{\mu_{0}}{\left[1+\theta\left(V_{G}-V_{T}\right)\right]^{2}}
$$

It is clear from the above that $\mu_{f e}$ is more sensitive to $\theta$ when compared with $\mu_{\text {eff. }}$ Therefore, $\mu_{f e}$ is often lower than $\mu_{\text {eff }}$ at higher $V_{G}$ values, as shown in Figure 3. ${ }^{[27]}$

The transconductance $g_{m}$ describes the ability of a transistor to modulate its output current upon application of a unit input voltage. Basically, $g_{m}$ is a device property rather than a physical parameter, which means that the $g_{m}$-based $\mu_{f e}$ may not be suitable for mobility characterization. One typical illustration of this point is that $g_{m}$ rises suddenly when the channel turns on, which does not reflect either the real mobility value or a mobility variation. ${ }^{[5,6]}$

\subsection{Saturation Mobility}

The saturation mobility is calculated from the saturation current:

$$
I_{\text {Dsat }}=\frac{W}{2 L} \mu_{\text {sat }} C_{i}\left(V_{G}-V_{T}\right)^{2}, \quad V_{D}>\left(V_{G}-V_{T}\right)
$$

where $I_{D s a t}$ is the saturation current and $\mu_{\text {sat }}$ is the saturation mobility.

In contrast to the mobilities derived from the output conductance or from the transconductance (which are in principle measured in the linear regime), the saturation mobility is measured in the saturation regime and has no explicit physical origin. High drain voltages or strong lateral fields can cause a series of problems, including velocity saturation ${ }^{[36]}$ and ambipolar conduction, ${ }^{[14]}$ that lead to underestimation or overestimation of the mobility. This problem will be discussed in more detail below.

\subsection{Hall Mobility}

The Hall mobility is not exactly a FET mobility because it is measured from specific samples by applying a magnetic field along with the applied electrical bias. Recently, however, use of the Hall mobility was proposed to characterize OFETs that showed severe nonidealities, or to confirm their high mobility and band-like charge transport. ${ }^{[4]}$ It is therefore necessary to review this mobility as well.

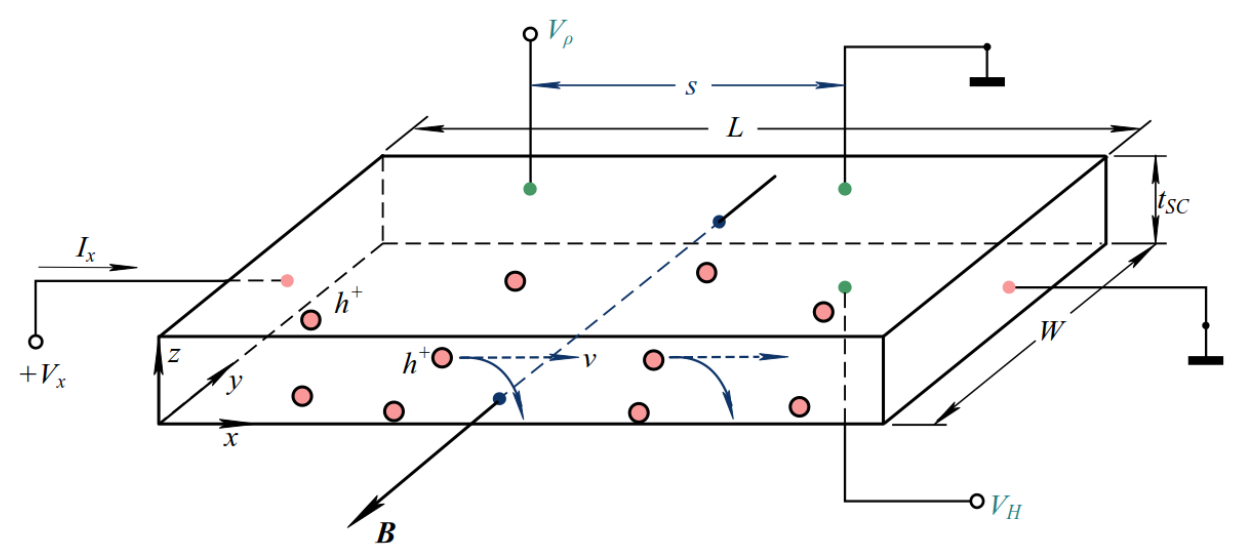

Figure 4. Illustration of Hall effect measurement on a transistor with a $p$-type semiconductor.

In the setup shown in Figure 4, the charges (holes) move from left to right under application of a lateral electric field $\left(E_{x}=V_{x} / L\right.$, where $V_{x}$ is the external bias and $L$ is the channel length). A magnetic field $B$ is applied that induces the Lorentz force to drive the holes to move downward toward the bottom of the structure, thus building a Hall voltage $V_{H}$ that is measured from the bottom toward the top of the semiconductor film. The Hall mobility $\mu_{H}$ is given by

$$
\mu_{H}=\frac{1}{q n} \frac{I_{x}}{V_{x}} \frac{L}{W t_{S C}}
$$

where $q$ is the elementary charge, $n$ is the charge concentration $\left(=I_{x} B / q W V_{H}\right.$, where $I_{x}$ is the external current and $W$ is the channel width), and $t_{S C}$ is the semiconductor layer thickness. Further details on Hall effect measurements can be found in the literature. ${ }^{[37-40]}$ 


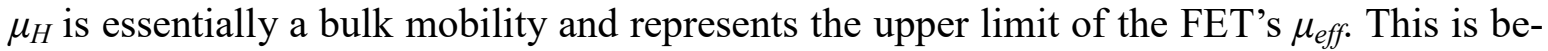
cause only mobile charges are driven by the Lorentz force to contribute to the Hall voltage while the trapped charges do not contribute. This can be understood in terms of the multiple trapping and release (MTR) model ${ }^{[41]}$

$$
\mu_{\text {eff }}=\mu_{0}{ }^{*} \frac{\tau}{\tau+\tau_{t r}}
$$

where $\mu_{0}{ }^{*}$ is the intrinsic trap-free mobility and $\tau$ is the average time required for a polaron to travel between shallow traps. Alternatively, this equation can be rewritten as ${ }^{[37]}$

$$
n_{\text {eff }}=n \frac{\tau}{\tau+\tau_{t r}}
$$

where $n_{\text {eff }}$ is the mobile charge density relative to the total charge density $n$. This observation implies that $\mu_{H}$ reflects the intrinsic trap-free mobility of a FET or a semiconductor and thus coincides with $\mu_{0}{ }^{*}$. Note that $\mu_{0}{ }^{*}$ here may not be equal to the low-field mobility $\mu_{0}$ that was discussed for FETs. Moreover, one may wonder that the trapped charges do not contributed to the channel current yet why they are sometimes taken into account when studying mobility. In fact, from a mobility extraction point of view, the effective mobility has reflected such a trapping effect since it value is lowered as the trap density increases.
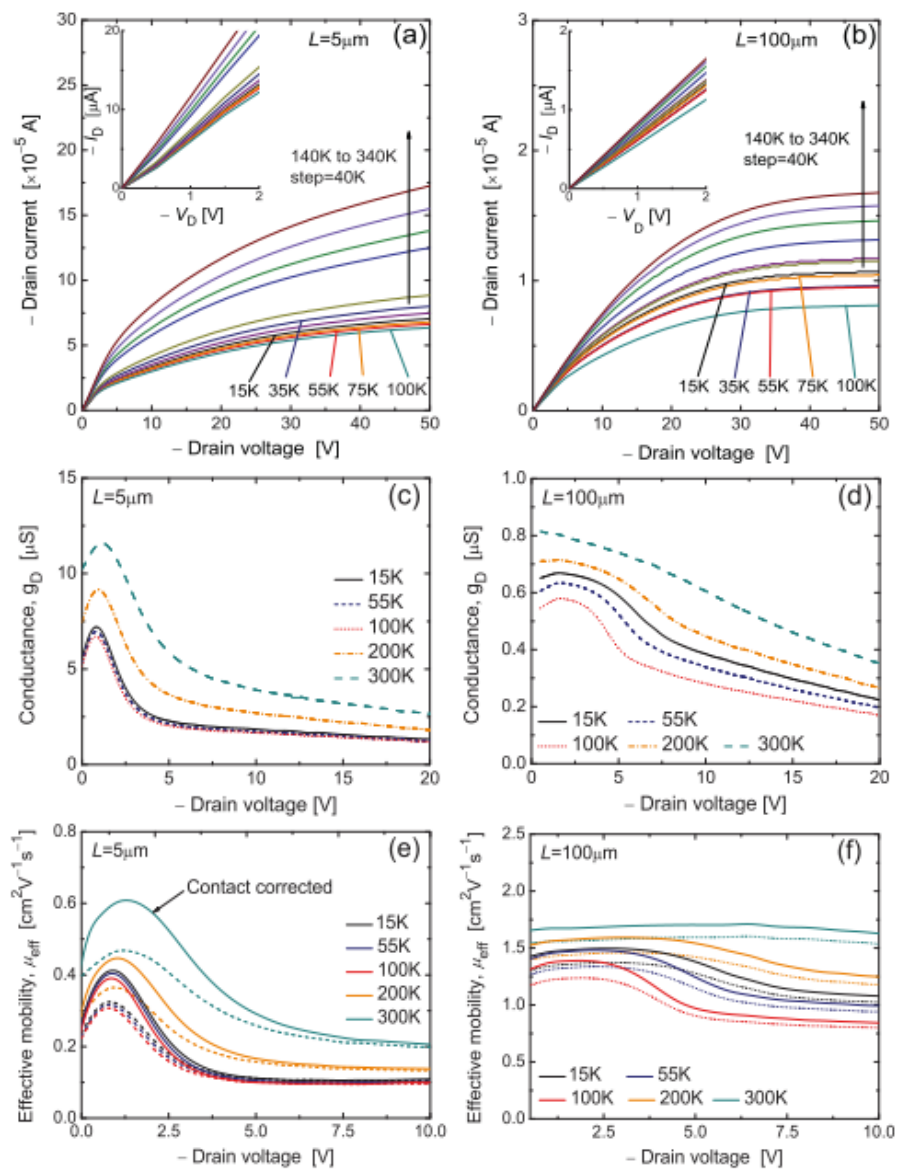

Figure 5. Extraction of effective mobility using the output conductance. Short-channel (left) and long-channel (right) 6,13-bis(triisopropylsilyl-ethynyl) pentacene (TIPS-pentacene) OFETs are measured at various temperatures. (a) and (b) illustrate their output characteristics at $V_{G}=-50 \mathrm{~V}$. The insets show the enlarged linear region in each case, where the short-channel device suffers from nonlinearity at low temperatures that is indicative of significant contact effects. (c) and (d) depict the deduced output conductance characteristics. (e) and (f) show the calculated effective mobility characteristics. Comparison shows that the room-temperature mobility in the longchannel device remains nearly constant with increasing drain voltage. ${ }^{[42]}$ Copyright 2011 , AIP.

\section{Extraction methods}

This section examines the corresponding methods required to extract the mobilities described above. 


\section{WILEY-VCH}

\subsection{Linear Output (LO) I-V Method to Extract the Effective Mobility}

The effective mobility $\mu_{\text {eff }}$ is deduced from the output conductance $g_{d}$. It is natural to measure the output characteristics $\left(I_{D}, V_{D}\right)$ and then use the linear region data ( $I_{D}$ before current saturation) to calculate $g_{d}=\partial I_{D} / \partial V_{D}$ from Equations (3) and (4); see Figure 5.

To extract $\mu_{e f f}$, it is necessary to determine $Q_{i}$, which is generally approximated using $C_{i}\left(V_{G}\right.$ - $V_{T}$ ), rather than use the $C$ - $V$ method because the $V_{G}$ is constant for each output curve. When the charge approximation applies, the data can be selected at a sufficiently high $V_{G}$ to ensure that the condition $V_{G} \gg V_{T}$ is satisfied. Otherwise, the channel is not in the strong accumulation regime. The real $Q_{i}$ can be lower than the value given by $C_{i}\left(V_{G}-V_{T}\right)$ and the mobility may be overestimated (similar to Figure 3 ).

Furthermore, $V_{D}$ may not be very small, e.g., $V_{D}$ can sweep up to $-5 \mathrm{~V}$ before current saturation. The maximum of $V_{D}=-5 \mathrm{~V}$ disturbs the charge uniformity from the source to the drain and is not negligible, even under the application of a high $V_{G}$. It is therefore necessary to include $V_{D}$ in the charge approximation. $\mu_{\text {eff }}$ is then extracted using

$$
\mu_{e f f}=\frac{L}{W} \frac{g_{d}}{C_{i}\left(V_{G}-V_{T}-V_{D}\right)}
$$

This method not only provides the mobility value but also offers the gate-voltage and drainvoltage dependences. Despite that, the prerequisite values of $C_{i}$ and $V_{T}$ are still required.

\subsection{Linear Transfer (LT) $I-V$ Method to Extract the Effective Mobility}

The transfer characteristics are used more frequently to extract the mobility when compared with the output characteristics. According to Equation (6), $I_{D l i n}$ shows linear variation with $V_{G}$, which means that $\mu_{\text {eff }}$ can be extracted via a linear fitting of $I_{\text {Dlin }}$ versus $V_{G}$, as shown in Figure 6a,

$$
\mu_{e f f}=\frac{L}{W} \frac{s_{1}}{C_{i} V_{D}}
$$

where $s_{1}$ is the slope of the linear fit.

(a)

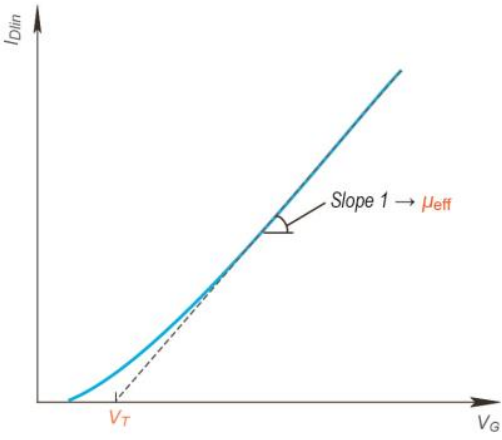

(b)

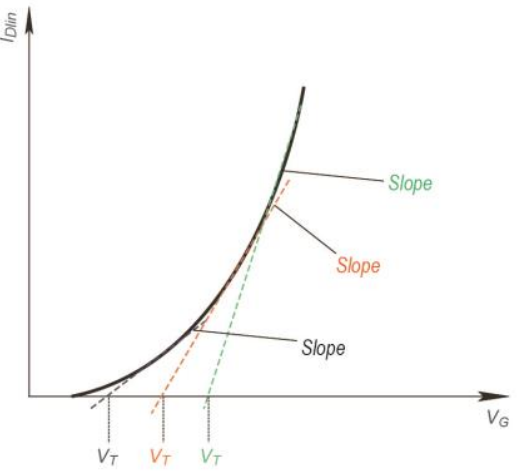

Figure 6. Illustration of the process of extraction of the effective mobility using the linear transfer data. (a) shows that the slope of the linear fitting gives the mobility, while the intercept at the $x$-axis gives the threshold voltage. (b) illustrates extractions with poor linearity. The different fittings produce very dissimilar mobilities and threshold voltages.

This method is based on the linear transfer data. The $I-V$ measurements should be implemented at a $V_{D}$ value as small as possible, while the linear fitting should be carried out at a $V_{G}$ that is high enough for the charge approximation hypothesis. Unlike the previous method, however, $V_{T}$ is not required. Instead, $V_{T}$ is often valued using the same linear fitting in combination with $\mu_{\text {eff }}$; see Figure 6a. Regardless of this, knowledge of $C_{i}$ remains a prerequisite.

The linear fitting essentially spans a wide range of data. If these data do not show good linearity, the mobility accuracy is strongly reliant on the fitting range (Figure 6b). ${ }^{[31]}$ It is known that $\mu_{\text {eff }}$ declines with increasing $V_{G}$ because of mobility attenuation (cf. Equation (8)). 


\section{WILEY-VCH}

The extraction of $\mu_{\text {eff }}$ is performed at low $V_{G}$, e.g., at a value around $V_{T}$, in an attempt to obtain the highest mobility. ${ }^{[5,6,39]}$ This is not appropriate when the charge approximation $Q_{i}=C_{i}\left(V_{G}\right.$ $\left.-V_{T}\right)$, which is valid only at $V_{G} \gg V_{T}$, is considered. In contrast, the high contact resistance plays the most important role at low $V_{G}$. A rapid drop in its value, e.g., due to lowering of the Schottky barrier, can cause a sharp increase in $I_{D}$, leading to artificially high mobility. ${ }^{[15]}$

\subsection{Linear Transfer (LT) $I-V$ Method Combined with $C$ - $V$ to Extract the Effective Mo- bility}

Introduction of the $C-V$ data eliminates the constraints and the uncertainty associated with the charge approximation. The $C-V$ measurements are implemented on metal-insulatorsemiconductor (MIS) capacitor structures with the same configuration as the OFETs to be characterized (including semiconductor and dielectric materials and processing conditions) or are implemented directly on the OFETs. The widely used metal-insulator-metal (MIM) capacitor structure, however, is not suitable for this purpose because it does not contain a semiconductor film and the measured results do not represent the OFET operational characteristics. For a more detailed discussion of the $C-V$ measurements, please refer to the literature. ${ }^{[13,26]}$

Using the $C-V$ data, $Q_{i}$ can be calculated by integrating $C_{G C}$ with respect to $V_{G}$ (cf. Equation (7) and Figure 2b) and then $\mu_{\text {eff }}$ can be computed as

$$
\mu_{\text {eff }}=\frac{L}{W} \frac{I_{D l i n}}{Q_{i} V_{D}}
$$

Note that the $I_{\text {Dlin }}$ value here is from the linear transfer data $\left(I_{D l i n}, V_{G}\right)$, with $V_{D}$ being a minor constant. This is quite different from the extraction approaches applied when using the output data. Additionally, the $C-V$ data $\left(C_{G C}, V_{G}\right)$ basically coincide with the OFET's linear transfer characteristics because the source and drain are tied together (in the OFET) or the back plate is grounded (in the MIS capacitor), meaning that the charge distribution in the channel is uniform.

This direct and precise method for $Q_{i}$ acquisition avoids use of both $C_{i}$ and $V_{T}$. The extracted $\mu_{\text {eff }}$ is thus reliable. This has profound significance for the still relatively undeveloped OFETs because their parameters vary greatly from batch-to-batch and from device-to-device. In addition, $Q_{i}$ is measured over the entire range of $V_{G}$. This means that Equation (19) is applicable not only for high $V_{G}$ but also for low $V_{G}$ values. The previous concerns about selection of the fitting range are also removed and the exact variation of $\mu_{\text {eff }}$ versus $V_{G}$ can be plotted (similar to Figure 3).

\subsection{Transfer I-V Method to Extract the Field-Effect Mobility}

The field-effect mobility $\mu_{f e}$ is deduced from the transconductance $g_{m}=\partial I_{D} / \partial V_{G}$ and thus from the transfer characteristics.

When the transistor operates in the linear regime, $\mu_{f e}$ is computed simply using

$$
\mu_{f e}=\frac{L}{W} \frac{g_{m}}{C_{i} V_{D}}
$$

where $V_{D}$ is a minor constant, as illustrated in Figure 3.

When the transistor operates in the saturation regime, $I_{D s a t}$ is independent of $V_{D}$ and Equation (20) is no longer applicable. $g_{m}$ then becomes

$$
g_{m}=\left.\frac{\partial I_{D}}{\partial V_{G}}\right|_{V_{D}=\text { constant }}=\frac{W}{L} \mu_{f e}{ }^{*} C_{i}\left(V_{G}-V_{T}\right)
$$

where $\mu_{f e}{ }^{*}$ is the saturation field-effect mobility. If $\mu_{f e}{ }^{*}$ is constant, $g_{m}$ then increases linearly with increasing $V_{G} . \mu_{f e}{ }^{*}$ can then be extracted via a linear fitting of $g_{m}$ with respect to $V_{G}$ :

$$
\mu_{f e}{ }^{*}=\frac{L}{W} \frac{S_{2}}{C_{i}}
$$

where $s_{2}$ is the slope of the linear fitting.

Note the differences between $\mu_{f e}$ and $\mu_{f e}{ }^{*}$ shown above. The linear $\mu_{f e}$ value can be a variable over the entire $V_{G}$ range, whereas the saturation value $\mu_{f e}{ }^{*}$ is only supposed to be constant within the strong accumulation regime (at $V_{G}>V_{T}$ ); otherwise, its extraction is ambiguous. 


\section{WILEY-VCH}

In fact, the linear $\mu_{f e}$ is recognized as the field-effect mobility. The saturation $\mu_{f e}{ }^{*}$, in contrast, is rarely used because it shows the same behavior as $\mu_{\text {sat }}$. However, the extraction process for $\mu_{\text {sat }}$ is quite different from that proposed here and will be discussed below.

\subsection{Y Function Method (YFM) for Extraction of the Low-Field Mobility}

Based on the previous mobility definition, the low-field mobility $\mu_{0}$ is superior to the other mobilities in terms of its inherence and reliability. The $\mathrm{Y}$ function method was proposed to extract $\mu_{0} \cdot{ }^{[33]}$ Using the linear transfer data, $g_{m}=\partial I_{D} / \partial V_{G}$ can first be calculated and then the Y function can be computed (see Figure 7):

$$
Y=\frac{I_{D l i n}}{\sqrt{g_{m}}}=\sqrt{\frac{W}{L} \mu_{0} C_{i} V_{D}}\left(V_{G}-V_{T}\right)
$$

where Equations (8), (10) and (11) are involved. When a constant $\mu_{0}$ is assumed, the Y function should vary linearly with $V_{G}$. Therefore, $\mu_{0}$ is attained by performing the following linear fitting:

$$
\mu_{0}=\frac{L}{W} \frac{S_{3}^{2}}{C_{i} V_{D}}
$$

where $s_{3}$ is the slope of the linear fitting.

Another noteworthy feature is that the mobility attenuation factor $\theta$ disappears in the $\mathrm{Y}$ function, although $\theta$ is included at the beginning of the deduction. Therefore, the $\mu_{0}$ value extracted here is free from those extrinsic influences and, most importantly, free from the effects of the contact resistance. ${ }^{[27,42]}$ This inherently reliable $\mu_{0}$ can be extracted directly.
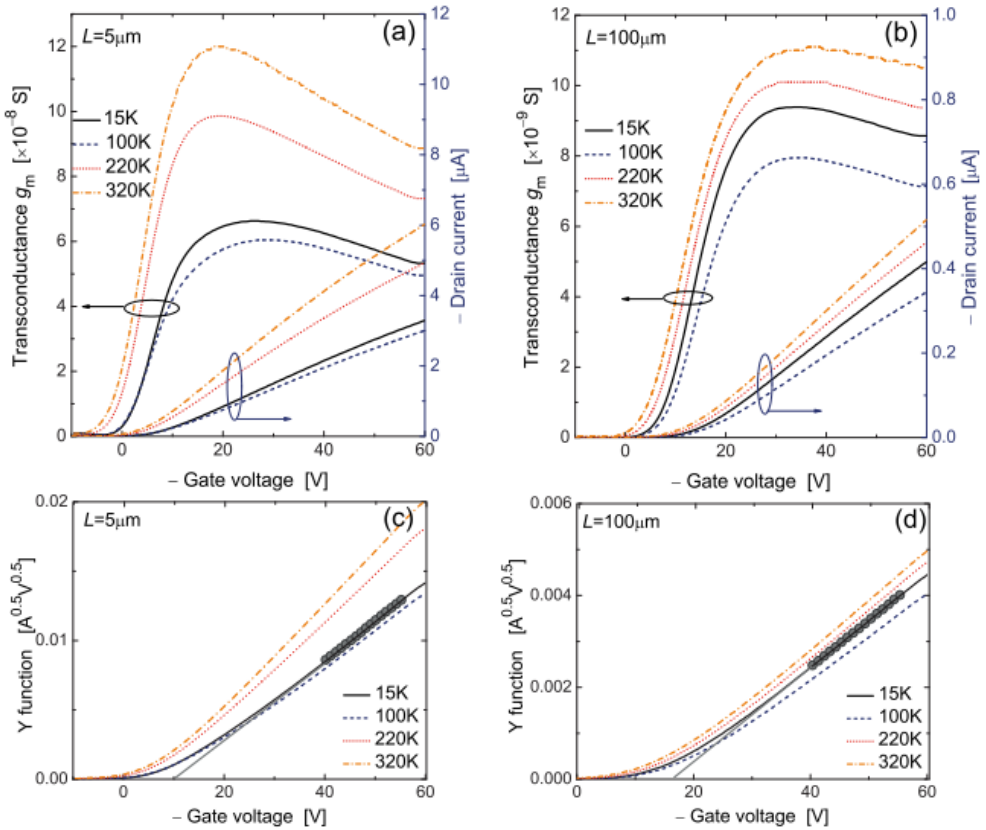

Figure 7. Extraction of low-field mobility using the $\mathrm{Y}$ function method. The two devices are those shown in Figure 5. (a) and (b) show the linear transfer curves and calculated transconductance characteristics for the shortchannel and long-channel TIPS-pentacene OFETs, respectively. (c) and (d) depict their calculated Y functions, which are highly linear, even when measured for the short-channel OFET at the ultra-low temperature of 15 K. ${ }^{[42]}$ Copyright 2011, AIP. 


\section{WILEY-VCH}
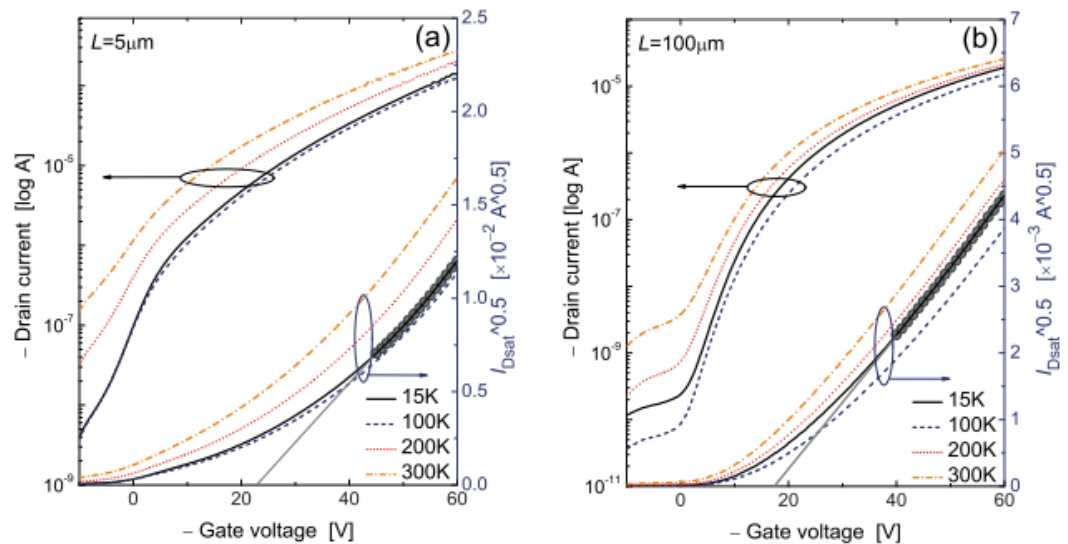

Figure 8. Extraction of the saturation mobility. The two devices used are those illustrated in Figure 5. (a) and (b) show the saturation transfer curve and the square root of the drain current that is fitted to extract the mobility. Note that the linearity of the results is poor in the short-channel device, particularly at low temperatures. ${ }^{[42]}$ Copyright 2011, AIP.

\subsection{Saturation Transfer (ST) $I-V$ Method to Extract the Saturation Mobility}

When a transistor operates in the saturation regime, its drain current $I_{D s a t}$ typically shows parabolic growth with increasing $V_{G}$. For extraction of the mobility, it is common to calculate the square root of $I_{D s a t}$ :

$$
\sqrt{I_{\text {Dsat }}}=\sqrt{\frac{W}{2 L} \mu_{\text {sat }} C_{i}}\left(V_{G}-V_{T}\right)
$$

The resulting $I_{\text {Dsat }}^{0.5}$ increases linearly with increasing $V_{G}$ and the saturation mobility $\mu_{\text {sat }}$ is then extracted by linear fitting (see Figure 8):

$$
\mu_{\text {sat }}=\frac{2 L}{W} \frac{S_{4}^{2}}{C_{i}}
$$

where $s_{4}$ is the slope of the linear fitting.

This method has been the most widely adopted to date in the organic electronics field as a consequence of its many trade-offs. For example, the saturation $I_{D s a t}$ is much higher than the linear $I_{D l i n}$, thus reducing the effects of the gate leakage. In some extreme cases, the leakage current $I_{G}$ can be even higher than $I_{D l i n}$. The measured net $I_{D}\left(=I_{D l i n}-I_{G}\right)$ does not increase but tends to decrease with increasing $V_{G}$, resulting in an erroneous mobility being obtained. The saturation regime is thus the only option. Additionally, the relatively high $V_{D}$ weakens the mobility attenuation of the contact resistance. A small value of $V_{D}=-1 \mathrm{~V}$ for the linear regime may dissipate by a substantial proportion at the contacts (e.g., $-0.7 \mathrm{~V}$ ), while the higher $V_{D}=-70 \mathrm{~V}$ for the saturation regime only loses a small percentage of its value. The underestimation of the relevant mobility can be alleviated. Therefore, this saturation-regime method is preferred because it offers feasible extraction and higher mobility.
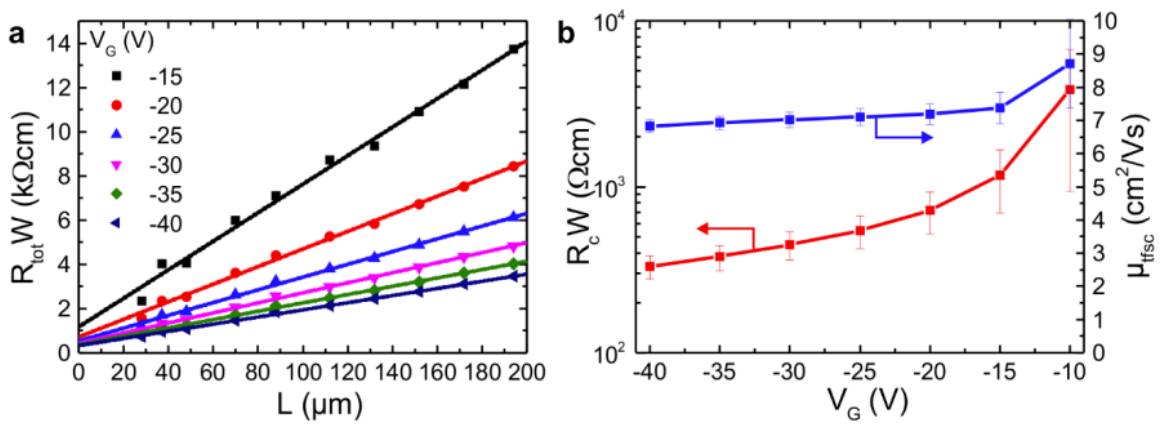

Figure 9. Extraction of the contact resistance and the mobility by the transmission-line method (TLM). (a) TLM plots for various gate voltages, where the contact resistance is determined from the $y$-axis intercept and the effective mobility is estimated from the slope of the linear regression plot. (b) Extracted results (where $\mu_{t f s c}$ is the effective mobility for a FET). ${ }^{[43]}$ Copyright 2016, Macmillan. 


\section{WILEY-VCH}

\subsection{Transmission-Line Method (TLM) for Extraction of the Effective Mobility}

The transmission-line method (TLM) is known for its use in extraction of the contact resistance. ${ }^{[4,45]}$ However, the TLM can also offer the effective mobility $\mu_{\text {eff }}$, as illustrated in Figure 9. ${ }^{[4]}$ The channel resistance of a transistor operating in the linear regime can be written as

$$
R_{\text {channel }}=\frac{V_{D}}{I_{\text {Dlin }}}=\frac{L}{W \mu_{e f f} C_{i}\left(V_{G}-V_{T}\right)}
$$

When the contact resistance $R_{C}$ is taken into account, the total resistance $R_{\text {total }}=R_{\text {channel }}+R_{C}$, i.e.,

$$
R_{\text {total }}=\frac{L}{W \mu_{e f f} C_{i}\left(V_{G}-V_{T}\right)}+R_{C}
$$

After measurement of a set of transistors with a diverse range of channel lengths, $R_{\text {total }}$ versus $L$ can be plotted and $R_{C}$ can be extracted from the $y$-axis intercept. Furthermore, the slope of the linear regression indicates $\mu_{e f f}$, which is given by

$$
\mu_{\text {eff }}=\frac{s_{5}}{W C_{i}\left(V_{G}-V_{T}\right)}
$$

where $s_{5}$ is the slope of the linear regression for a given value of $V_{G}$. When $V_{G}$ is varied, the gate-voltage dependence of $\mu_{\text {eff }}$ can also be acquired.

In practice, each linear regression is based on a set of $R_{\text {total }}$ values deduced from the transfer data at a common $V_{G}$. The linearity may deteriorate if $V_{T}$ varies greatly from device to device. The incorporation of $V_{T}$ is essential to improve the linearity and in turn to enhance the reliability of the mobility. ${ }^{[46]}$

\subsection{Gated Four-Point Probe (gFPP) Method to Extract the Effective Mobility}

Similar to the TLM, the gFPP method provides $\mu_{\text {eff }}$ without the contact effect, in addition to its more common use for contact resistance evaluation. However, when compared with the TLM, which only requires conventional FETs, the gFPP necessitates the fabrication of two extra probes between the source and the drain that are separated by a distance $D$, as shown in Figure 10. Along with their measurement of the linear transfer characteristics, the two probes can detect the potential drop $\Delta V$, which allows $\mu_{\text {eff }}$ to be extracted using

$$
\mu_{\text {eff }}=\frac{D}{W C_{i} \Delta V} \frac{d I_{D l i n}}{d V_{G}}
$$




\section{WILEY-VCH}

(a)

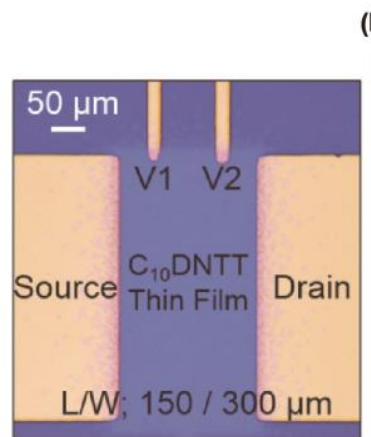

(c)

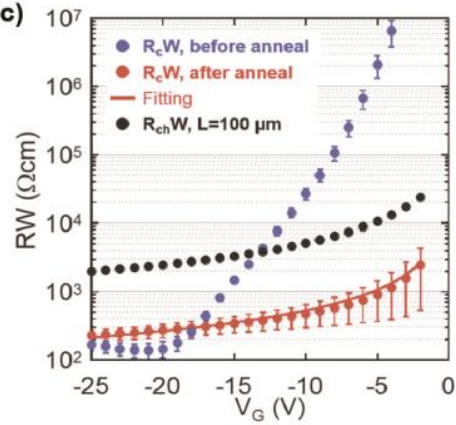

(b)

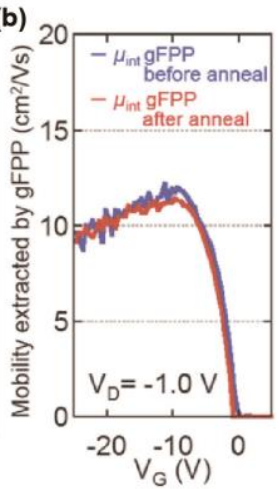

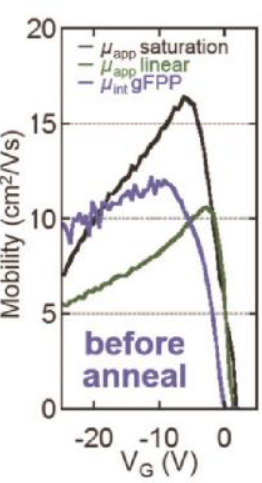

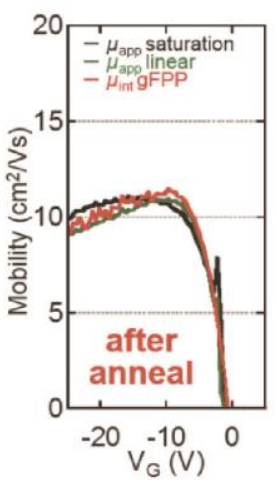

(d)
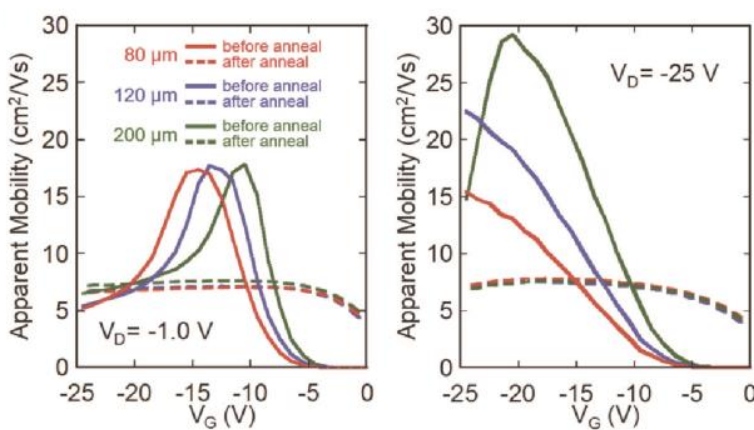

Figure 10. Extraction of the effective mobility using the gFPP method. (a) Optical microscopic image of a 2,9didecyl-dinaphtho[2,3-b:2', $3^{\prime}$-f] thieno[3,2-b]-thiophene $\left(\mathrm{C}_{10}\right.$-DNTT) OFET with two additional probes fabricated between the source and drain electrodes. (b) Left: Mobility as measured using the gFPP method. Middle and Right: illustrations of the mobilities extracted by different methods from the nonannealed and annealed devices, respectively. Note that the mobilities coincide after annealing and approach the mobility characteristics extracted by the gFPP method even before annealing, as shown in the left panel, thus indicating that the gFPP method provides mobility values that are free from the contact effect. (c) Contact resistance vs. gate voltage. (d) Mobility characteristics extracted from the linear (left) and saturation (right) regimes. ${ }^{[5]}$ Copyright 2015, Wiley.

Uemura et al. investigated $\mathrm{C}_{10}$-DNTT OFETs using the gFPP method (Figure 10a). ${ }^{[5]}$ They found that devices without annealing suffered from high contact resistance (Figure 10c), which caused large variations in the transconductance. The conventional transfer $I-V$ methods always overestimated the mobility (Figure 10b, middle panel). After annealing, the contact resistance was reduced but the device characteristics were retained and this provided a much more reliable mobility (Figure 10b, right panel). The overestimation was greater in the longerchannel devices measured in the saturation regime (Figure 10d), with a maximum of 30 $\mathrm{cm}^{2} \mathrm{~V}^{-1} \mathrm{~s}^{-1}$ (even reaching up to $100 \mathrm{~cm}^{2} \mathrm{~V}^{-1} \mathrm{~s}^{-1}$ ), while the intrinsic mobility was only approximately $7 \mathrm{~cm}^{2} \mathrm{~V}^{-1} \mathrm{~s}^{-1}$. Interestingly, the mobility extracted using the gFPP method was found to be independent of such effects (Figure 10b, left panel) and was sufficiently reliable for characterization of new materials and technologies.

While it allows the intrinsic, contact-free $\mu_{\text {eff }}$ to be extracted, this method has several limitations. First, the additional probes increase the device fabrication complexity. Second, these probes may not collect the exact potential drop in the channel because they can disturb the potential distribution if they are not of a negligible size and their contact with the semiconductor is not ohmic. In addition, they may not be located directly in the channel; e.g., in top-gate, bottom-contact devices, the channel is located close to the top surface of the semiconductor film but these extra probes are placed at the bottom of the semiconductor film. The error can be amplified as the OSC film thickness increases. The applicable device configurations are thus restricted by these problems.

\subsection{Hall Effect Method for Extraction of the Hall Mobility}




\section{WILEY-VCH}

The Hall mobility is basically measured from specimens such as a Hall bar and a van der Pauw sample. If the measurement equipment includes a four-point probe, the Hall mobility $\mu_{H}$ can be also measured from a FET, as studied by Podzorov et al. (see Figure 11), using ${ }^{[37]}$

$$
\mu_{H}=\frac{1}{B} \frac{V_{H}}{V} \frac{L^{*}}{W}
$$

where $V$ is the voltage applied across the two extra contacts between the source and the drain, and $L^{*}$ is the distance between these two extra contacts.

Similar to the gFPP method, Hall effect measurements are not readily accessible either. These measurements require additional fabrication for two extra probes and a strong magnetic field is required to enhance the Hall voltage because the OSC mobility is usually very low. Hall effect measurements on organic devices often suffer from high noise and the Hall mobility may not be always accessible.

(a)

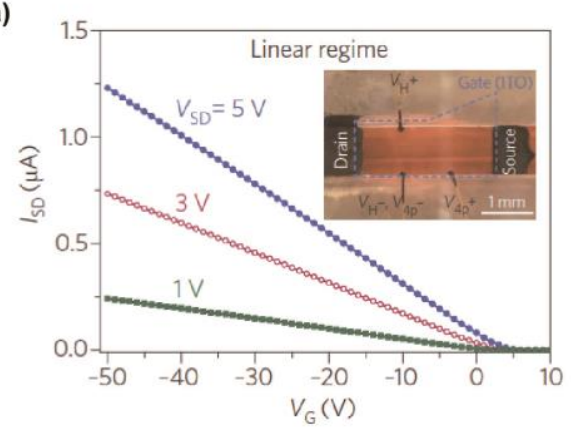

(b)

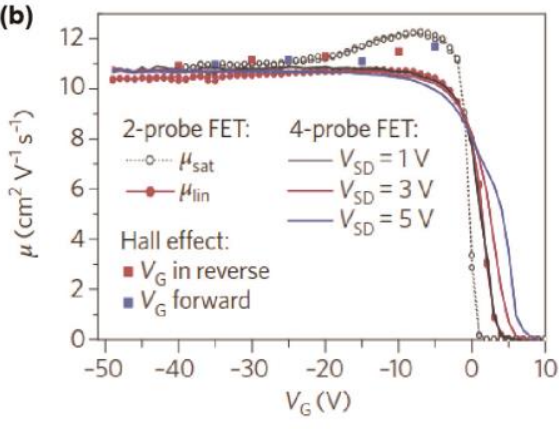

Fig. 11 Illustration of mobility extraction via Hall effect measurements. (a) Linear transfer curves from a singlecrystal rubrene OFET (inset) with extra probes that are used to measure the potential difference and the Hall voltage. (b) Mobilities extracted via the conventional method (two-probe FET), the gFPP method (four-probe FET) and the Hall effect. Because of the near-ideal device characteristics, these mobilities are very close, which means that mobility extraction reliability can even be high for the conventional method. ${ }^{[4]}$ Copyright 2018 , Macmillan.

At the end of this section, it must be emphasized that linear fitting and differentiation are sometimes interchangeable in these procedures. $\mu_{\text {eff }}$ can be extracted via linear fitting of $I_{\text {Dlin }}$ over a wide range of high $V_{G}$ values (see Figure 3 and Equation 18), yet it is also possible to differentiate $I_{\text {Dlin }}$ with respect to $V_{G}$ in the strong accumulation regime to plot $\mu_{\text {eff }}$, as illustrated in Figure 3. In short, linear fitting assumes constant mobility, which means that a wide bias range for mobility extraction is feasible. Otherwise, the fitting range is ambiguous and the extracted mobility may then be unreliable. The differentiation procedure that is obligatory for calculation of the output conductance and the transconductance assumes variable mobility from the beginning, which means that the bias dependences are yielded directly. However, it remains essential to take the criteria for selection of the raw data for each method into account, as summarized in Table 1. Additionally, linear fitting remains prevalent at present because of its ease of use when reporting on mobility. As a result, Choi et al. recently proposed the factor $\gamma$ to gauge the reliability of mobility extraction when using linear fitting. ${ }^{[4]}$

\section{Device Fabrication Considerations}

To extract the mobility with high precision, many factors must be taken into consideration during device fabrication. This section addresses a few of these factors that are becoming serious concerns in this field.

\subsection{Gate Leakage Control}

The on-state current of a transistor is a manifestation of charge drift (perhaps with a minor diffusion contribution). In the presence of gate leakage, some of the charges deviate from the 


\section{WILEY-VCH}

channel under attraction by the gate field and flow out through the gate dielectric; see Figure 12a. The net output current to be measured is lower than it should be. Mobility underestimation is then inevitable and higher leakage leads to increasingly serious mobility underestimation. In some extreme cases, the leakage is so high that the leaked charges grow more rapidly than the charges injected from the source. ${ }^{[47]}$ The drain current is turned to decrease with increasing gate voltage, which makes mobility extraction impossible (Figure 12b). This explains why extraction of the mobility in the saturation regime has been so popular, because it partially circumvents this leakage problem.

(a)

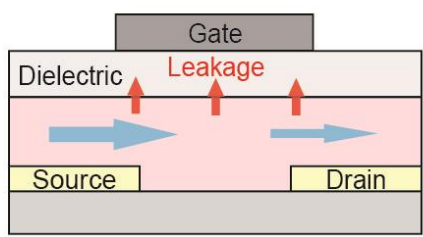

(c)

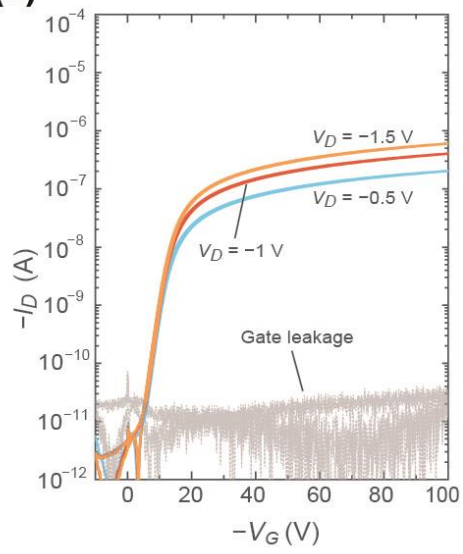

(b)

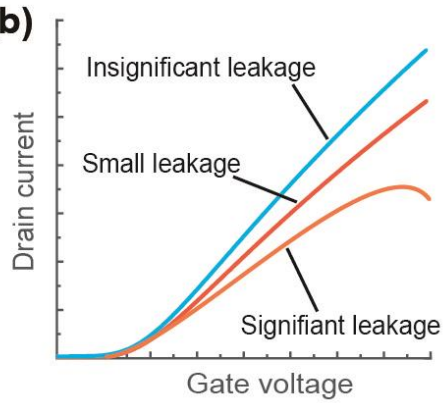

(d)

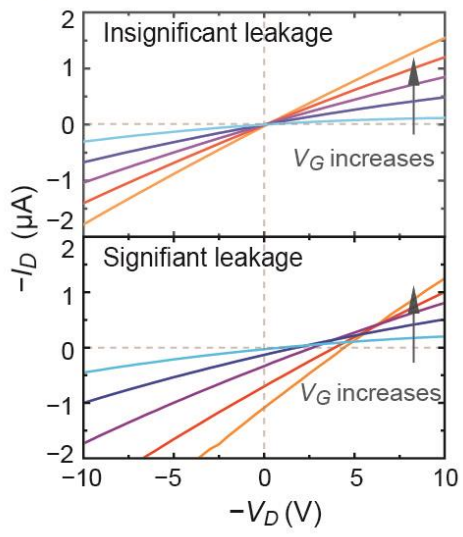

Figure 12. Gate leakage. (a) Illustration of the leakage mechanisms in a FET. The net drain current can be reduced as a result of charge losses caused by the gate leakage. (b) Illustration of gate leakage in the transfer curves. Significant leakage can notably reduce the current growth rate, thus causing mobility underestimation. (c) Observation of gate leakage in the transfer curves by direct measurement of the leakage current. ${ }^{[15]}$ Copyright 2017, Wiley. (d) Indirect observation of gate leakage using the output characteristics. In the case of more significant leakage, the drain current consistently shifts downward as the gate voltage increases.

Gate leakage can be observed directly when measuring the transfer curve, as illustrated in Figure 12c. It is also possible to observe the gate leakage indirectly using the output characteristics. Figure 12d shows that if the output curves pass through the origin, the leakage is insignificant. Otherwise, the output curves shift downward at $V_{D}=0 \mathrm{~V}$, which is indicative of significant leakage because a zero $V_{D}$ should impose a zero $I_{D}$, but the gate leakage is mainly dependent on $V_{G}$. Thus, as $V_{G}$ increases, a more negative (or opposite) $I_{D}$ is observed at $V_{D}=0$ $\mathrm{V}$ and this is entirely due to the gate leakage.

Suppression of the gate leakage can be achieved using robust dielectrics with superior dielectric performance, thicker films, and even multilayer structures. ${ }^{[48]}$ In addition, patterning of the OSC film is indispensable. This is particularly important for solution-processed OFETs with bottom-gate architectures, e.g., $\mathrm{Si} / \mathrm{SiO}_{2}$ structures where the $\mathrm{Si}$ and $\mathrm{SiO}_{2}$ layers serve as the common gate and the common gate dielectric, respectively. Application of a sufficiently high $V_{G}$ turns the entire OSC film into an active area. However, if the OSC film is not well isolated from the back Si gate (e.g., at the substrate edge), the leakage problem will be severe. Patterning of the OSC film cuts off the electrical connection between the gate and the active area and the current flow is confined within a small region; see Figure 13. 


\section{WILEY-VCH}

(a) Without OSC film patterning

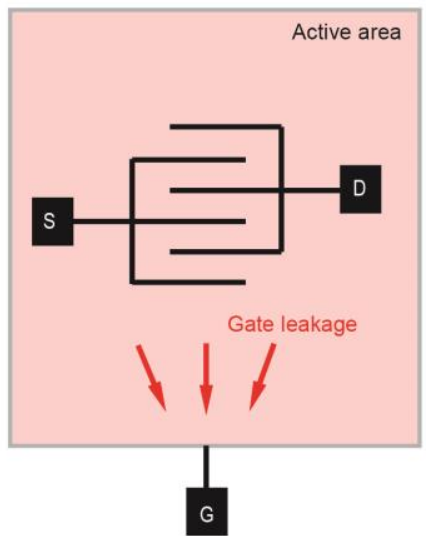

(b) After inappropriate patterning

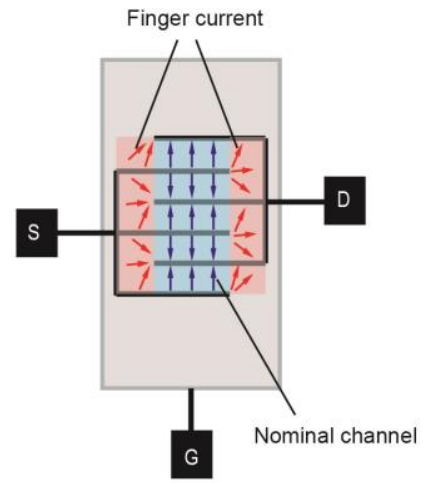

(c) After appropriate patterning

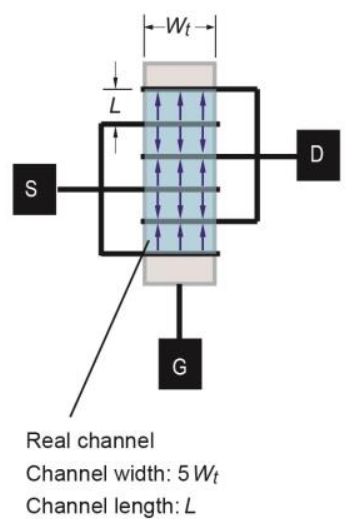

Figure 13. Illustration of OSC patterning to suppress gate leakage and finger currents. (a) A nonpatterned OSC film is turned into an active area (red zone) under application of a sufficiently high gate voltage. The leakage current can then pass through the conductive OSC film and flow toward the gate via the substrate edge. (b) An OSC film that is inappropriately patterned because finger currents (red zone) still exist outside the overlapped channel (blue area). (c) When the OSC film has been patterned to be entirely within the overlapped region, the finger current is suppressed and the channel dimensions can be defined explicitly.

\subsection{Calibration of the Channel Dimensions}

Calibrated channel dimensions are a primary requirement for mobility extraction. While the technological dimensions can be precisely measured via microscopy, the real, effective channel width and length may still differ. As illustrated in Figure 13b, finger currents exist outside the overlapped interdigital electrodes if the OSC film is not patterned appropriately. Because the overlapping area defines the nominal channel, the parasitic finger currents alter the effective channel width and length, e.g., doubling the channel width, which leads to double overestimation of the mobility. This issue will worsen as the device becomes increasingly miniaturized. Patterning of the OSC film and/or reduction of the gate coverage area to limit the current flow such that it occurs precisely within the region with explicit channel definition is the only way to eliminate the finger currents, as shown in Figure 13c.

\subsection{Consideration of the Channel Length}

To date, there is still no consensus with regard to the channel length required to guarantee reliable mobility extraction. In general, it is better to use long-channel devices for the following reasons.

First, in bottom-contact OFETs, the presence of the contact electrodes disrupts the molecular self-organization during OSC film deposition, producing high-density defects in the vicinity of the contact, which sometimes appear as small-sized grains, in contrast to the large-sized grains present in the central channel. This defect-rich zone is rather wide (up to tens of micrometers ${ }^{[27]}$ and can extend further into the channel as the contact thickness increases and/or the pristine grain size grows. ${ }^{[49]}$ The overall charge transport may then be dominated completely by the charge trapping and hopping at the contacts and this was observed in highly crystalline 6,13-bis(triisopropylsilyl-ethynyl) pentacene (TIPS-pentacene) OFETs, where the mobility rose constantly with increasing $L$, whereas the correlated activation energy declined with increasing $L .^{\left[{ }^{42]}\right.}$ After $L$ exceeded $100 \mu \mathrm{m}$, the activation energy appeared to saturate, which implied that such long-channel OFETs were almost immune to contact effects and were thus reliable choices for mobility evaluation and charge transport analysis. ${ }^{[42]}$

Second, the leakage current between the source and drain electrodes becomes noticeable as $L$ continues to shrink ${ }^{[50,51]}$ because of the absence of doping and the accumulation-regime operation of the device. ${ }^{[52]}$ This parasitic leakage increases the off-state current, particularly in 


\section{WILEY-VCH}

the saturation regime and can be regarded as a type of short-channel characteristic. If $L$ is not long enough to suppress this leakage, then the on-state current can be also affected. Consequently, the mobility can be overestimated under these conditions.

Third, the velocity saturation is serious in very short-channel devices. When this saturation occurs, the carrier velocity no longer grows with the lateral field induced by $V_{D}$. Much earlier current saturation (theoretically at $V_{D}=\left(V_{G}-V_{T}\right)$ ) can be observed in the output characteristics. ${ }^{[36]}$ This implies that the measured $I_{D}$ is lower than it should be and thus the mobility is underestimated in this case. This issue mainly affects the saturation regime. The linear regime, upon application of a minor $V_{D}$, is largely safe in this respect.

\subsection{Basic Optimization}

Because the FET mobility involves numerous extrinsic effects, basic device optimization is required to preclude any significant effects on the device charge transport. Refined molecular design and/or polymer-small molecule blending enhance the charge transport capability of the FET and minimize the energetic disorder, ${ }^{[1,7,53,54]}$ suitable deposition techniques such as barcoating and off-center spin-coating improve the molecular arrangement and thus reduce the structural defects, ${ }^{[55-57]}$ appropriate surface treatments such as use of a self-assembled monolayer (SAM) reduce the surface roughness scattering and the trap density; ${ }^{[58,59]}$ use of low- $\kappa$ dielectric materials weakens the energetic disorder imposed by the incorporated dipoles; ${ }^{[60-63]}$ appropriate contact engineering to reduce the Schottky barrier and/or to fabricate nonrectifying contacts will also minimize the contact effects. ${ }^{[11,64,65]}$ After these steps, the device will demonstrate near-ideal FET characteristics that will allow reliable mobility extraction to be performed.

\section{Calibration of the Specific Capacitance of the Gate Dielectric}

Examination of Table 1 indicates that $C_{i}$ is a prerequisite for all mobility extraction methods, except for the approach that combined $C$ - $V$ and Hall effect measurements. $C_{i}$ determines the charge density in the channel, meaning that if it is underestimated, the mobility will then be proportionally overestimated. In theory, $C_{i}$ is defined as

$$
C_{i}=\frac{\varepsilon_{0} \varepsilon_{S}}{t_{i}}
$$

where $\varepsilon_{0}$ is the vacuum permittivity, $\varepsilon_{S}$ is the relative permittivity of the gate dielectric, and $t_{i}$ is the dielectric film thickness. $C_{i}$ can be calculated using the nominal dielectric constant and thickness, but their values in practice may differ greatly. To provide the mobility precisely, it is better for $C_{i}$ to be measured experimentally.

Recently, Xu et al. discussed two approaches using MIS and OFET structures to measure $C_{i}$, i.e., using $C$ - $V$ methods (Figure 14). ${ }^{[13]}$ MIS capacitors can be fabricated with identical configurations to that of the OFET to be characterized (including the dielectric and semiconductor materials and the processing conditions) to reflect the OFET $C$ - $V$ characteristics consistently. The advantage of the MIS structure over the OFET is that the MIS capacitor can be prepared at a much larger size to allow the device signal-to-noise ratio to be improved. In addition, the MIS structure is less sensitive to the AC signal frequency, particularly when the OSC mobility is low. However, the OFET is readily accessible without extra device fabrication requirements.

In the same work by $\mathrm{Xu}$ et al., the frequency effect was also investigated. When compared with conventional Si MOSFETs that use high-frequency signals of up to $1 \mathrm{MHz}$, OFETs require signals at much lower frequencies because of the low mobility in the OSC. At very high frequencies, the charge carriers may not be able to follow the AC signal to allow them to be redistributed over the (active) OSC film because the measured capacitance is normalized with respect to the overlapped plate area or the channel area, thus leading to an underestimated $C_{i}$. Strictly speaking, the $C$ - $V$ measurements in this case must be performed under either static or 


\section{WILEY-VCH}

quasi-static conditions because the $I-V$ data that are characterized by the parameter analyzer are basically static and the $C-V$ data should therefore also be similarly static. In practice, use of several low frequencies, e.g., $20 \mathrm{~Hz}, 50 \mathrm{~Hz}, 100 \mathrm{~Hz}$, may be attempted or capacitancefrequency $(C-f)$ measurements may be implemented under application of a sufficiently high bias to confirm that the frequency dependence is not involved (see Figure 14). Otherwise, use of quasi-static $C-V$ measurements or similar static techniques is obligatory. ${ }^{[6,31]}$ Unfortunately, the mobility overestimation caused by this type of $C_{i}$ underestimation has lasted for years. In some extreme cases, the level of overestimation can exceed orders of magnitude. Measures must be implemented to control this problem.

In addition to $C_{i}$ determination, the measured $C$ - $V$ data can also be used to calculate $Q_{i}$ (see Equation (7) and Figure 2). Using this precise $Q_{i}$, it is possible to extract the effective mobility directly over the entire gate voltage range without the need to calibrate $C_{i}$ (Equation (19)).

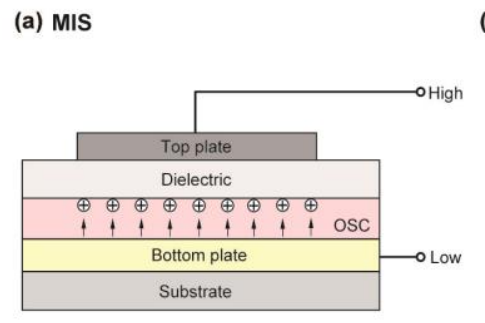

(b) OFET

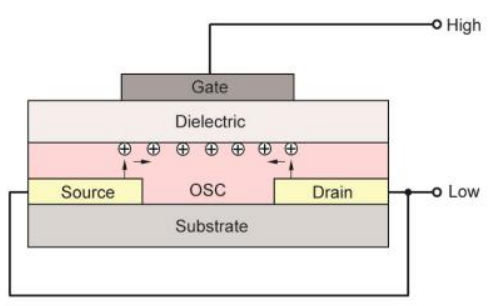

(c)

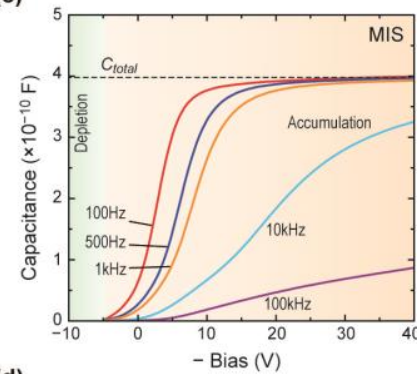

(d)

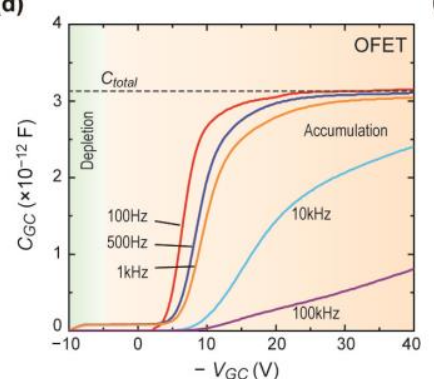

(e)

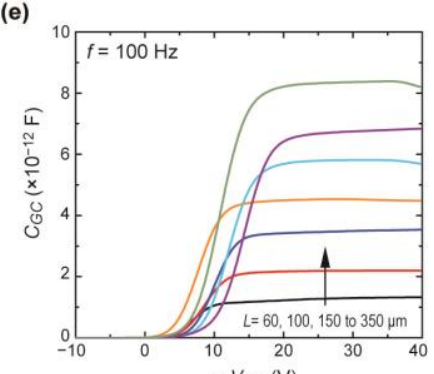

(f)

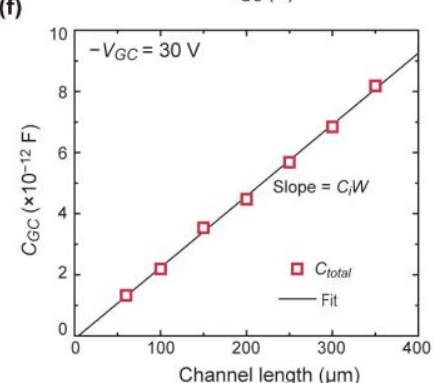

Figure 14. Illustration of the $C-V$ measurement processes. (a) and (b) show the device structures of the MIS device and the OFET, respectively, where the measurement setup and the charge accumulation processes are depicted. (c) and (d) show typical $C-V$ results measured at various frequencies for the MIS device and the OFET, respectively. (e) and (f) illustrate a channel length scaling procedure used to improve the accuracy of extraction of $C_{i} \cdot{ }^{[13]}$ Copyright 2018, Wiley.

\section{Calibration of the Threshold Voltage}

After $C_{i}$, knowledge of the threshold voltage $V_{T}$ is the second prerequisite for mobility extraction (see Table 1). Incorporation of $V_{T}$ is essential for the mobility reliability factor $\gamma$ proposed by Choi et al. The reliability is solely dependent on the data linearity and should not be affected by $V_{T}$. The inclusion of $V_{T}$ eliminates its side effects and thus the revised $\gamma$ can serve as a fair indicator of the reliability of the mobility. ${ }^{[4,13]}$
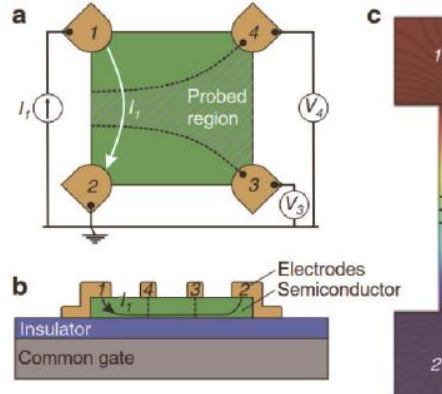

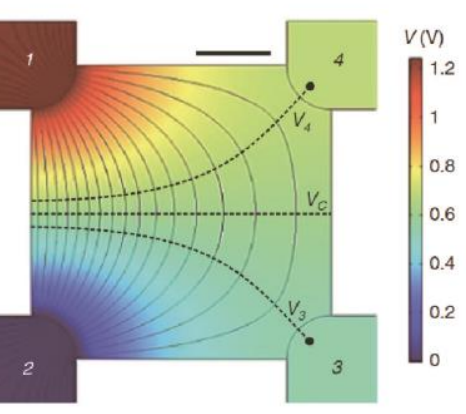

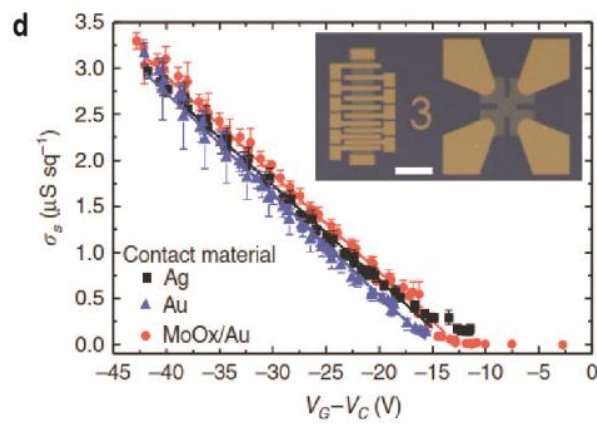

Figure 15. Illustration of the mobility and threshold voltage extraction processes using the gated van der Pauw (gVDP) method. (a) and (b) illustrate the device structure and measurement setup. (c) depicts the potential map 


\section{WILEY-VCH}

and shows the current density within the semiconductor film. (d) shows the measured conductivity vs. gate voltage characteristics for devices with different contact materials. ${ }^{[43]}$ Copyright 2017, Macmillan.

The $V_{T}$ in OFETs is complicated by the different operating principles of these devices and the presence of extrinsic effects (e.g., charge trapping). ${ }^{[28,52]}$ Precise measurement of the $V_{T}$ is difficult for OFETs. Nevertheless, it is believed that good linearity indicates the validity of the applied model. $\mathrm{Xu}$ et al. found that the $\mathrm{Y}$ function consistently demonstrated great linearity in the face of a number of extrinsic effects, indicating its versatility for $V_{T}$ extraction. ${ }^{[13]}$ Additionally, accurate $V_{T}$ values can be determined using advanced techniques such as $C-V^{[32]}$ and transconductance derivative methods. ${ }^{[26,66]}$ Recently, Rolin et al. developed the gated van der Pauw (gVDP) method to extract an OSC mobility that was free from contact effects (see Figure 15). ${ }^{[3]}$ Interestingly, this method delivered a precise $V_{T}$ regardless of the contact metals used, which is actually quite significant for mobility extraction.

\section{Contact Effects}

The contact resistance $R_{C}$ has long been the most important factor affecting mobility extraction. In OFETs, $R_{C}$ comprises two components: the interface resistance $R_{\text {interface }}$ and the bulk resistance $R_{b u l k}$. The former component originates from charge injection at the metalsemiconductor interface and is mainly governed by the Schottky barrier (see Figure 16a). ${ }^{[64]}$ The latter component arises from the access transport through the OSC bulk with relatively low charge concentrations and perhaps with an inferior charge transport profile (e.g., caused by contact defects). ${ }^{[67-69]}$ Together, these resistances alter the device characteristics, making mobility extraction unreliable or even impossible.

(a)

Conventional polymer transistor

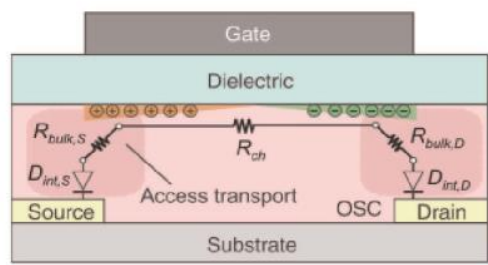

(d)

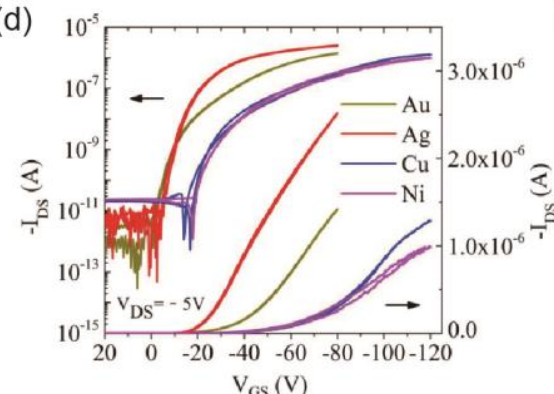

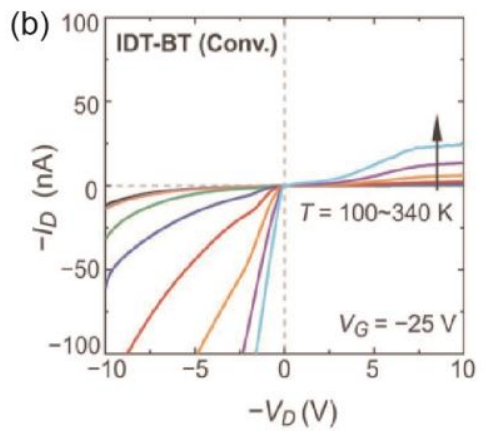

(e)

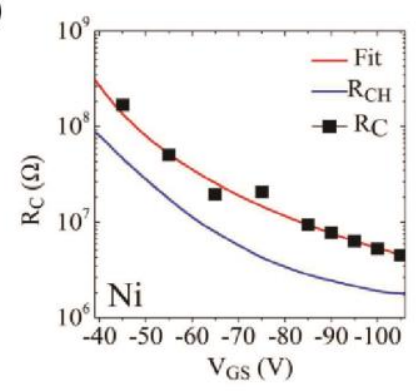

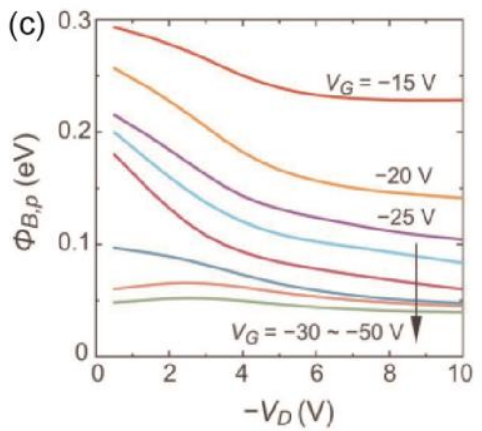

(f)

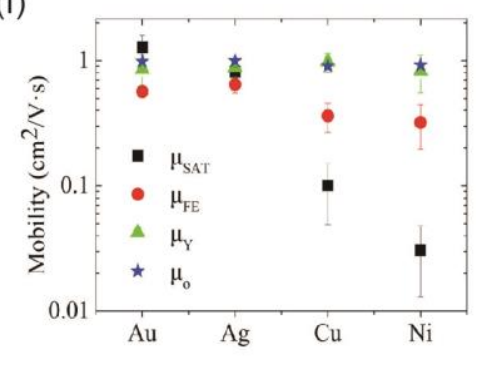

Figure 16. Contact effects in OFETs. (a) Typical device structure of state-of-the-art polymer transistor, in which the contact resistance stems from interface charge injection and the access transport through the OSC bulk. (b) Measurement of the Schottky barrier of an indacenodithiophene-co-benzothiadiazole (IDT-BT) OFET. (c) Schottky barrier measured at various drain voltages and gate voltages. ${ }^{[15]}$ Copyright 2017, Wiley. (d) Linear transfer curves of a set of IDT-BT OFETs using various contact metals. (e) Comparison of the contact resistance and the channel resistance in an OFET with Ni contacts. (f) Summary of the mobilities extracted using the different methods. Only the low-field mobility extracted via the Y function method is almost independent of the contact metal used. ${ }^{[70]}$ Copyright 2019, IEEE.

$\mathrm{Xu}$ et al. investigated indacenodithiophene-co-benzothiadiazole (IDT-BT) OFETs and found that the Schottky barrier in these devices ranged up to $0.64 \mathrm{eV}$, although this value de- 


\section{WILEY-VCH}

clined rapidly to $0.24 \mathrm{eV}$ upon sweeping of $V_{G}$ from $0 \mathrm{~V}$ to $-20 \mathrm{~V}$ (Figure $16 \mathrm{~b}$ and 16c). ${ }^{[15]}$ The resulting $R_{\text {interface }}$ value can drop by a tremendous factor of $5.4 \times 10^{6}$ (that may even be amplified to $1.2 \times 10^{10}$ at the low temperature of $200 \mathrm{~K}$ ), which is much greater than the decline in the channel resistance $\left(\sim 10^{4}\right)$. This result implies that the apparent reduction in the total resistance (i.e., the increase in the drain current) is dominated by the very high and rapidly decreasing $R_{\text {interface }}$. While this contact feature does not represent the real channel behavior, its rapid change means that it cannot be used to characterize the mobility. In fact, several other reports have pointed out the serious mobility overestimation that can be caused in this way. ${ }^{[5}$, 6]

Unlike $R_{\text {interface }}$, which mainly affects the low $V_{G}$-regime, $R_{\text {bulk }}$ can play a major role at higher $V_{G}$ values. This is because the channel resistance undergoes a fast decline during channel formation, whereas $R_{C}$ cannot. $^{[68]}$ The total resistance is dominated by $R_{C}$ and the apparent mobility is then limited. This issue is particularly significant for short-channel OFETs measured in the linear regime. To remedy this effect, the measured $I_{D}$ can be corrected using a preknown $R_{C}$ (evaluated via the Y function method or the TLM). ${ }^{[27]}$

$$
I_{D c o r}=\frac{I_{D}}{1-\left(\frac{I_{D}}{V_{D}}\right) R_{C}}
$$

where $I_{D c o r}$ is the $R_{C}$-corrected drain current. This contact-resistance-free $I_{D c o r}$ permits reliable mobility extraction, even when using conventional methods.

In practical situations, $R_{C}$ is unknown before the measurements. More advanced techniques are desired to measure the mobility without the contact effect. The YFM, the TLM, the gFPP method and the Hall effect method are all suitable methods; among these methods, the YFM is the easiest to use. The YFM requires only a single, normal OFET and the measurement of a linear transfer curve. The intrinsic, contact-free low-field mobility can then be extracted directly. As shown in Figure 16d, e and f, Huang et al. found that their IDT-BT OFETs with Ni contacts suffered from high contact resistance but the YFM was still able to measure the mobility reliably. ${ }^{[70]}$ Despite these results, basic contact optimization is required anyway to reduce both the Schottky barrier and the contact charge trapping. ${ }^{[45]}$ Here, it is interesting to note that the sharp turning on/off caused by the largely varied Schottky barrier is appealing for making high-gain, ultra-low power devices and circuits, as demonstrated by Nathan's group at Cambridge. ${ }^{[71,72]}$

\section{Bias Dependences}

Mobility extraction using linear fitting assumes a constant mobility, at least within the fitting range. However, in most cases, this assumption may not be satisfied. The mobility can vary significantly with the application of external biases.

\subsection{Gate-Voltage Dependence}

For a bulk OSC with uniform charge concentration $n$, the mobility can be regarded as being constant. As $n$ increases (e.g., upon doping), the effective mobility $\mu_{\text {eff }}$ varies depending on the density of states (DOS) profile of the OSC; see Figure 17a. When the DOS is full of delocalized states, $\mu_{\text {eff }}$ is high and declines with increasing $n$, which is indicative of degeneracy (Figure 17b). The charge transport is band-like. For a DOS that includes localized states (mostly presented as tail states), $\mu_{\text {eff }}$ is low and is positively dependent on $n$. The charge transport mainly occurs by hopping at low $n$ and the delocalization then improves at higher $n$. If the energetic disorder rises, the weight of hopping is also augmented. As a result, $\mu_{\text {eff }}$ is further reduced and becomes more positively $n$-dependent. ${ }^{[42]}$

In a FET, $V_{G}$ causes more charges to be distributed near the dielectric-semiconductor interface. The charge concentration $n$ within the OSC film decays exponentially from the interface toward the bulk and can be averaged over the entire thickness to be equivalent to the charge 


\section{WILEY-VCH}

density per unit area (Figure 17c). The $\mu_{\text {eff }}$ that was discussed above certainly varies among the different thicknesses and was finally averaged by solving Poisson's equation to determine an apparent $\mu_{\text {eff. }}$ This apparent $\mu_{\text {eff }}$ is the effective mobility that is often measured from OFETs. As $V_{G}$ increases, the $n$ distribution in the OSC film evolves from a more uniform distribution in the weak accumulation regime to a superficial distribution in the strong accumulation regime. The dependence of $\mu_{\text {eff }}$ on $V_{G}$ is actually quite complex ${ }^{[73]}$ but it can still be investigated via calculations. As shown in Figure $17 \mathrm{~d}$, Xu et al. observed that fully band-like transport (where $\alpha=1$ ) gives a high $\mu_{\text {eff }}$ that constantly decreases with increasing $V_{G}{ }^{\left[{ }^{[7]}\right.}$ In contrast, hopping in the localized states (for lower $\alpha$ ) with greater energetic disorder (higher $\Delta E$ ) causes $\mu_{\text {eff }}$ to degrade by more than two orders of magnitude, while $\mu_{\text {eff }}$ changes into a $V_{G}$-enhanced quantity. Increased hopping and disorder lead to a lower $\mu_{\text {eff }}$ and greater $V_{G}$-enhancement also occurs. In fact, this strong $V_{G}$-enhancement is unwanted for mobility extraction because it causes the data to become superlinear, as observed in the OFETs with high-density defects. ${ }^{[31 \text {, }}$ 42]

When the surface roughness scattering, the contact resistance, and other factors are taken into account, the $V_{G}$-dependence can become more complex. In general, a higher $V_{G}$ value attracts the charges towards the interface, thus raising these surface effects. In addition, the contact resistance may begin to dominate the overall resistance. The relevant $\mu_{\text {eff }}$ is also degraded by the higher $V_{G}$. This negative $V_{G}$-dependence counterbalances the positive dependence discussed above. The apparent outcome is dependent on the relative impacts of these dependences. Note that the contact resistance, and particularly the interface resistance caused by the high Schottky barrier, can notably increase the $V_{G}$-enhancement (Figure 16c); however, such increases mainly occur at small values of $V_{G}$.

(a)

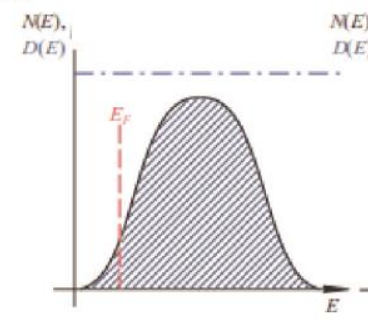

(b)
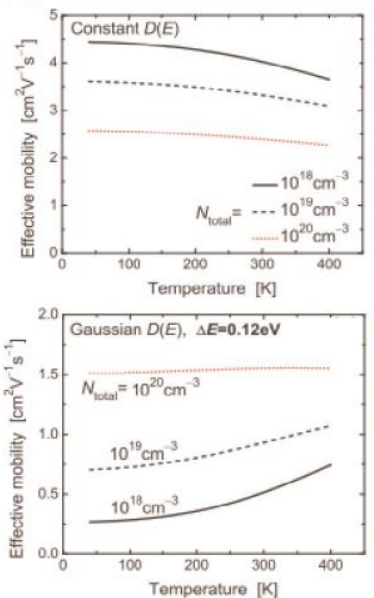
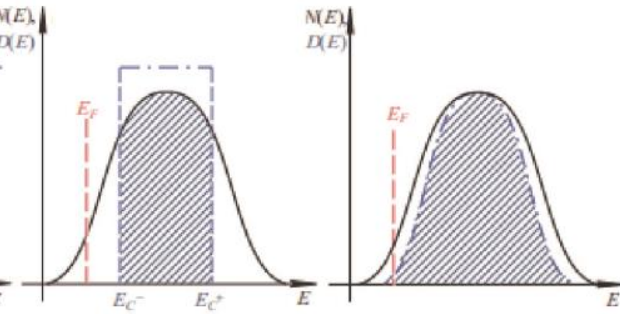

(c)

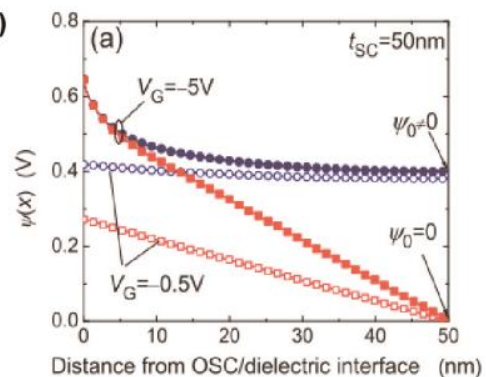

(d)
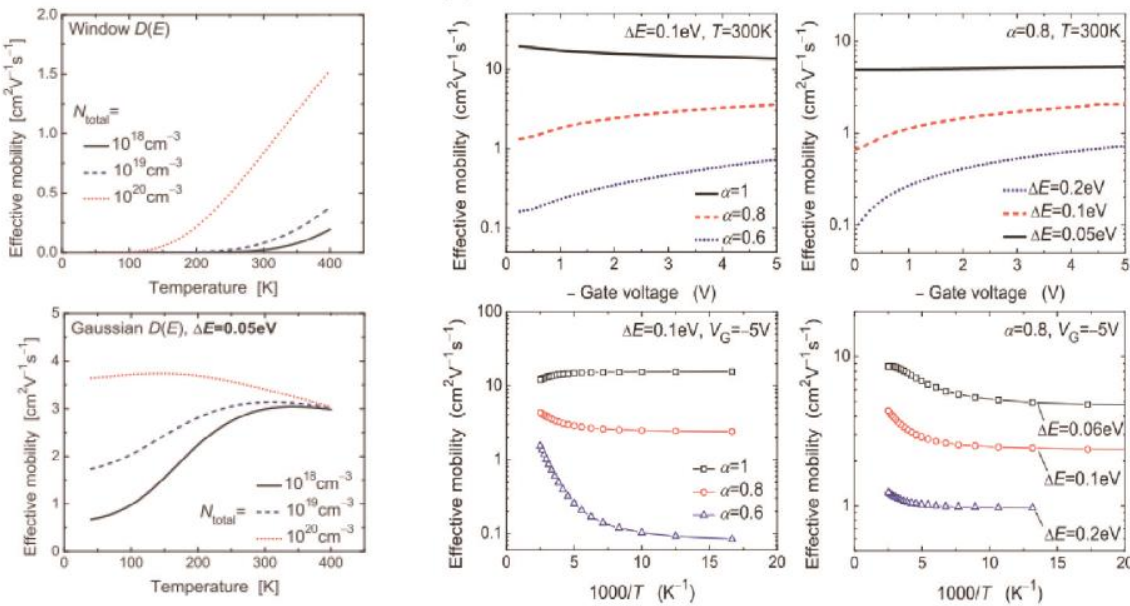

Figure 17. Gate-voltage dependence of the mobility. (a) Three different DOS profiles with constant diffusivity (left), a window diffusivity distribution (middle), and a Gaussian diffusivity distribution (right). (b) Calculated mobility vs. temperature at various charge concentrations for the three DOS profiles. ${ }^{[42]}$ Copyright 2011, AIP. (c) Simulated potential profile in an OFET with a $50 \mathrm{~nm}$ pentacene film. When a sufficiently high gate voltage is applied, the potential shifts greatly on the left surface, thus indicating charge accumulation at that surface. (d) Calculated mobility for this OFET vs. gate voltage and temperature. ${ }^{[74]}$ Copyright 2011 , AIP. 


\section{WILEY-VCH}

Because the $V_{G}$-dependence is very complex and difficult to predict, it is better to perform the mobility extraction by differentiation. If linear fitting is mandatory, then selection of an appropriate method is vital. Examination of Table 1 shows that only the Y function method would be suitable for this purpose. The mobility attenuation factor $\theta$ actually lumps all the $V_{G^{-}}$ dependences together at the beginning, while $\theta$ is ultimately eliminated. The slope of the $\mathrm{Y}$ function is highly linear, indicating a constant $\mu_{0}$ that is independent of $V_{G}$. Therefore, the extraction of $\mu_{0}$ using linear fitting over a wide $V_{G}$ range is feasible. ${ }^{[13]}$

\subsection{Drain-Voltage Dependence}

The drain voltage $V_{D}$ plays a relatively less important role in altering the mobility when compared with $V_{G}$. As $V_{D}$ increases, the charge distribution in the channel no longer remains uniform. After the channel is pinched off at the drain, the charge carriers located there must pass through a depletion region with a transport profile that would be inferior to that of the channel. The mobility can then decrease with increasing $V_{D}$, which would manifest as a declining saturation current in the output characteristics. ${ }^{[75]}$ In contrast, the lateral field induced by $V_{D}$ helps to lower the Schottky barrier (see Figure 16c). The mobility can be increased by $V_{D}$, which would be exhibited as $V_{D^{-}}$enhancement. ${ }^{[76]}$ This explains in part why mobility extraction in the linear regime is not preferred because the mobility obtained would be relatively low.

(a)

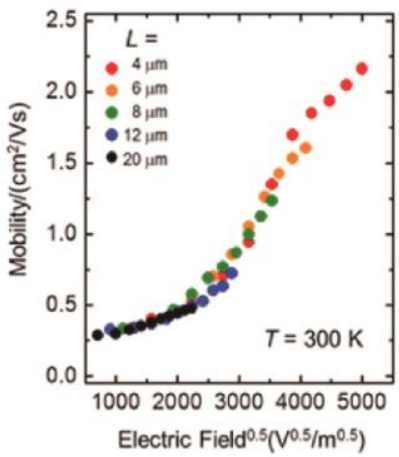

(d)

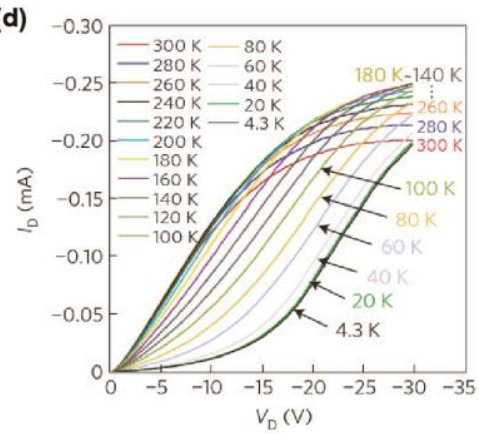

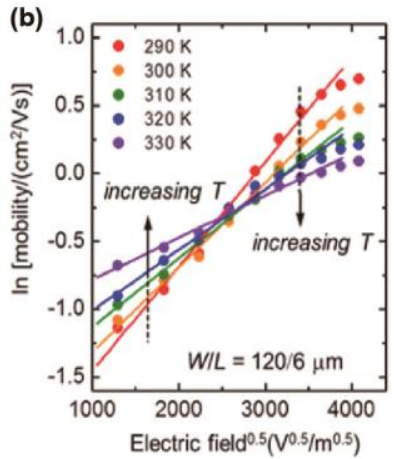

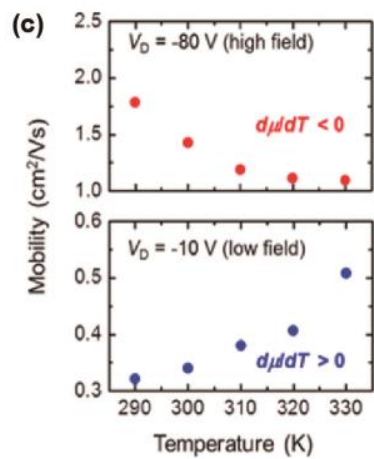

(e)

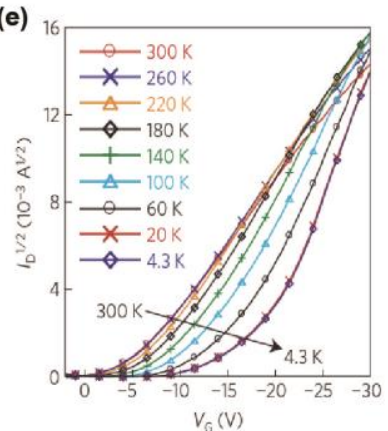

(f)

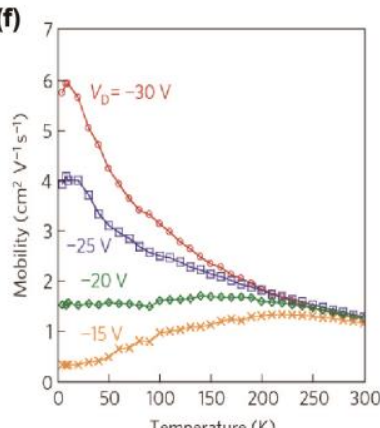

Figure 18. Drain-voltage dependence of the mobility. (a) Mobility measured under various lateral electric fields. (b) Mobility plotted vs. the square root of the lateral electric field at different temperatures. (c) Temperature dependence of the mobility under low and high lateral electric fields. ${ }^{[76]}$ Copyright 2015, ACS. (d) Output characteristics of a TIPS-pentacene OFET measured at various temperatures. (e) Square root of the saturation current plotted vs. gate voltage for mobility extraction. The curve clearly shifts downward upon cooling, while the linearity also worsens. (f) Saturation mobility vs. temperature at various drain voltages. ${ }^{[77]}$ Copyright 2010, Macmillan.

With regard to mobility extraction, the $V_{D^{-}}$dependency can be observed by considering the output conductance characteristics; see Figure 5. However, this dependence is limited to the linear regime (i.e., at small $V_{D}$ ). When a very high $V_{D}$ is applied, several issues may arise.

i) Ambipolar conduction can be triggered if the charge injection barriers to the holes and electrons are not high enough to block one of these carriers. The two types of charge carriers 


\section{WILEY-VCH}

would combine to contribute to the drain current and the mobility would inevitably be overestimated. More importantly, it is difficult to discriminate how much the two carrier types contribute separately. As $V_{G}$ increases, the charge transport first occurs in an ambipolar regime and eventually enters the unipolar regime (see Figure 1d, right panel). A mobility peak often appears between these regimes. This means that only the last data measured at the highest $V_{G}$ are reliable in terms of avoidance of ambipolar effects. ${ }^{[14]}$

ii) A strong lateral field changes the potential landscape for the charge transport by lowering the hopping barrier. ${ }^{[15]}$ The mobility value is thus improved and the relevant activation energy is reduced. In some cases, this effect is so significant that the hopping barrier seems to vanish and band-like charge transport is then observed (Figure 18). ${ }^{[76,77]}$ While these high mobility, low activation energy, and band-like transport characteristics may be appealing, they may not represent the intrinsic characteristics of the OSCs and OFETs to be characterized.

iii) When the lateral field becomes comparable to the vertical gate field, the off-state current may be greatly increased. ${ }^{[50]}$ This changes the device characteristics (e.g., the threshold voltage) and thus affects mobility extraction.

iv) A strong lateral field may also cause velocity saturation, particularly for very shortchannel devices, that would lead to underestimation of the mobility.

v) A high $V_{D}$ also imposes a strong $I_{D}$, which means that the current density is high. This imposes another problem in that the Joule heating can become severe in some volumes, e.g., in the OSC bulk at the contacts. ${ }^{[67]}$ Because the OSC's thermal conductivity is generally very low, the heated OSC may experience high-temperature localized annealing and may even be burned if the high-power-density measurements continue. The electrical properties of the OSC may be substantially degraded, causing nonideal device characteristics and leading in turn to unreliable mobility extraction. This is why pioneering researchers recently suggested a thermal budget control approach based on estimation of the maximum current density and the maximum power density while also quoting the measurement timescale. ${ }^{[4]}$

Overall, it is better to extract the mobility in the linear regime.

\section{Temperature Dependence}

In addition to the mobility value, the temperature dependence of the mobility is also important because it has typically been used to probe the charge transport. ${ }^{[7,15,78,79]}$ For example, the positive temperature dependence (i.e., the thermal activation) is regarded as an indicator of hopping conduction. The relevant activation energy characterizes the averaged hopping barrier. $^{[15,78,80]}$ In contrast, the negative temperature dependence is believed to be a characteristic of band-like charge transport. ${ }^{[77,81,82]}$ Unreliable mobility extraction can lead to large deviations in the temperature dependence characteristics. ${ }^{[83]}$ 

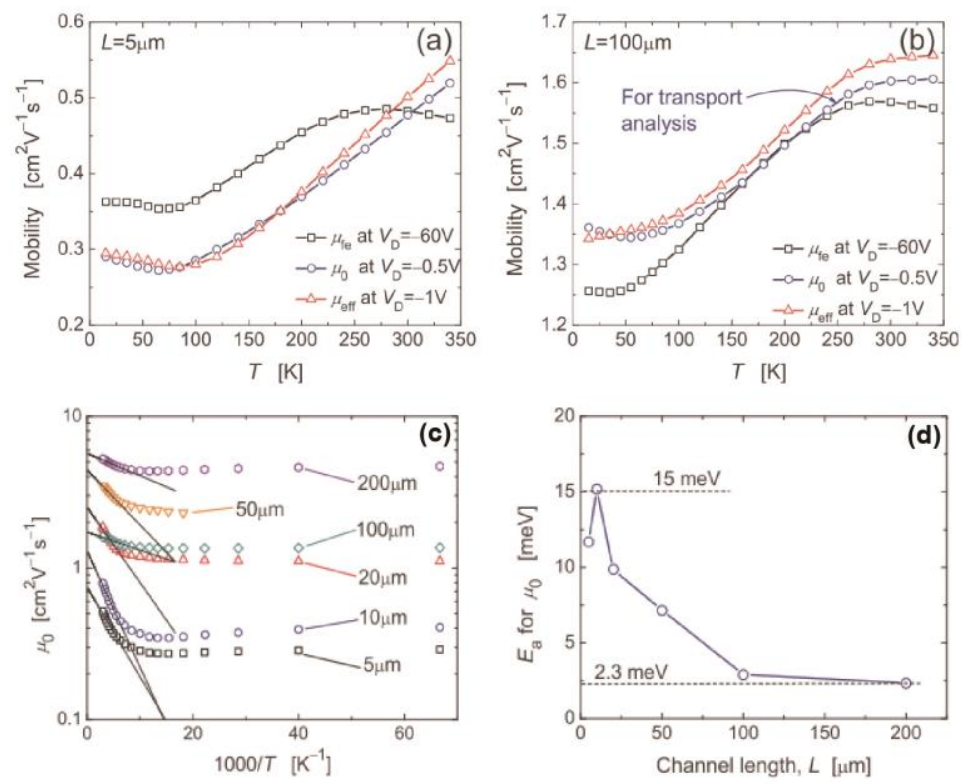

Figure 19. Temperature dependence of the mobility. (a) and (b) show the mobilities that were extracted from short-channel and long-channel TIPS-pentacene OFETs, respectively. The figures show that the low-field mobility extracted via the $\mathrm{Y}$ function method and the effective mobility extracted via the output conductance are very close, while the saturation mobility always deviates. (c) Arrhenius plots for OFETs with various channel lengths. (d) Activation energy vs. channel length characteristics. ${ }^{[42]}$ Copyright 2011, AIP.

$\mathrm{Xu}$ et al. inspected three different mobilities in polycrystalline TIPS-pentacene OFETs (Figure 19) and found that the commonly used saturation mobility $\mu_{\text {sat }}$ always deviated from both $\mu_{\text {eff }}$ and $\mu_{0}$, whereas the latter two remained very close. ${ }^{[42]}$ The deviations were reflected in terms of the mobility value and the temperature dependence. Note that $\mu_{\text {sat }}$ shows a negative temperature dependence at high $T$, whereas the other two mobilities do not. This implies that $\mu_{\text {sat }}$ may mistakenly point toward band-like transport within a small temperature range. Comparison of the short- $L$ and long- $L$ OFETs reveals that contact effects make $\mu_{\text {sat }}$ artificially higher than both $\mu_{\text {eff }}$ and $\mu_{0}{ }^{[5]}$ Therefore, the consistent $\mu_{\text {eff }}$ and $\mu_{0}$ values are more reliable as compared with $\mu_{\text {sat }}$. Nevertheless, the temperature dependences of $\mu_{0}$ (and $\left.\mu_{\text {eff }}\right)$ still differ between the short $L$ and long- $L$ OFETs. Beyond $T>100 \mathrm{~K}$, the $\mu_{0}$ of the short- $L$ OFET consistently shows thermal activation while its long- $L$ counterpart tends to saturate at room temperature. Examination of OFETs with various $L$ values showed that the activation energy falls monotonically with increasing $L$, which is indicative of a diminishing contact effect. Interestingly, the contact conductance shows a very similar temperature dependence to that of $\mu_{0}$ in the short- $L$ OFET. Overall, these results showed that the charge transport in short- $L$ OFETs is dominated by the contact transport, which mostly involves hopping. The mobilities extracted from the affected OFETs, particularly $\mu_{\text {sat }}$, may lead to misinterpretation of the charge transport and thus mislead the subsequent research. This is one of the reasons why long- $L$ OFETs are more reliable for mobility extraction and charge transport analysis applications.

\section{Conclusion}

In conclusion, a review of the carrier mobility extraction for organic transistors has been presented. Unlike the recent literature, this article has focused on a precise extraction approach that is mainly based on the device current-voltage characteristics. The review began by defining the various mobilities and then moved on to the relevant extraction methods to ensure clear understanding of their origins, their physical meanings, and the limitations on data selection. After that, device fabrication and the prerequisite calibration processes were addressed, including leakage control, channel dimension calibration, channel length selection, 


\section{WILEY-VCH}

and calibration of the specific gate-dielectric capacitance and the threshold voltage, which have both been overlooked to date. Finally, the gate-voltage and drain-voltage dependences were examined along with the temperature dependence. While the TLM, the gFPP method and the Hall effect methods could provide reliable mobility, they either require additional device fabrication steps or necessitate the use of special instruments and thus are not readily accessible in typical fabrication facilities. The mostly widely used mobility, the saturation mobility, unfortunately may not be sufficiently accurate to evaluate the mobility value or to probe the charge transport. The low-field mobility that was extracted via the Y function method, however, demonstrated obvious advantages in terms of its reliability and robustness. For more precise mobility extraction, complementary $C-V$ measurements that give the specific gate-dielectric capacitance accurately are also still necessary.

\section{Acknowledgment}

We thank David MacDonald, MSc, from Liwen Bianji, Edanz Editing China (www.liwenbianji.cn/ac), for editing the English text of a draft of this manuscript. This work was financially supported by National Natural Science Foundation of China under grant Nos. 61804078, Natural Science Foundation of Jiangsu Province under grant Nos. BK20180759 and Start-Up Fund from Nanjing University of Posts and Telecommunications (NY218149) and also sponsored by NUPTSF (Grant No.NY219003). 


\section{WILEY-VCH}

Table 1: Summary of mobility extraction methods.

\begin{tabular}{|c|c|c|c|c|c|}
\hline Method & LO $I-V$ & LT $I-V$ & LT $I-V$ with $C-V$ & LT $I-V$ for $\mu_{f e}$ & ST $I-V$ for $\mu_{f e}$ \\
\hline Raw data & $\begin{array}{l}\text { Linear output } \\
\qquad\left(I_{D l i n}, V_{D}\right)\end{array}$ & $\begin{array}{l}\text { Linear transfer } \\
\quad\left(I_{\text {Dlin }}, V_{G}\right)\end{array}$ & $\begin{array}{l}\text { Linear transfer } \\
\quad\left(I_{\text {Dlin }}, V_{G}\right) \\
+C-V\left(C_{G C}, V_{G}\right) \\
\end{array}$ & $\begin{array}{l}\text { Linear transfer } \\
\quad\left(I_{\text {Dlin }}, V_{G}\right)\end{array}$ & $\begin{array}{l}\text { Saturation transfer } \\
\qquad\left(I_{D s a t}, V_{G}\right)\end{array}$ \\
\hline $\begin{array}{l}\text { Data selec- } \\
\quad \operatorname{tion}^{1}\end{array}$ & $\begin{array}{c}V_{D}<\left(V_{G}-V_{T}\right) \\
V_{G}>>V_{T}\end{array}$ & $\begin{array}{c}V_{D}<<\left(V_{G}-V_{T}\right) \\
V_{G}>>V_{T}\end{array}$ & $V_{D}<<\left(V_{G}-V_{T}\right)$ & $V_{D}<<\left(V_{G}-V_{T}\right)$ & $\begin{array}{c}V_{D}>\left(V_{G}-V_{T}\right) \\
V_{G}>>V_{T}\end{array}$ \\
\hline Prerequisite $^{2}$ & $C_{i}, V_{T}$ & $C_{i}$ & & $C_{i}$ & $C_{i}$ \\
\hline $\begin{array}{l}\text { Mobility to } \\
\text { extract }\end{array}$ & $\mu_{\text {eff }}$ & $\mu_{e f f}$ & $\mu_{e f f}$ & $\mu_{f e}$ & $\mu_{f e}{ }^{*}$ \\
\hline
\end{tabular}

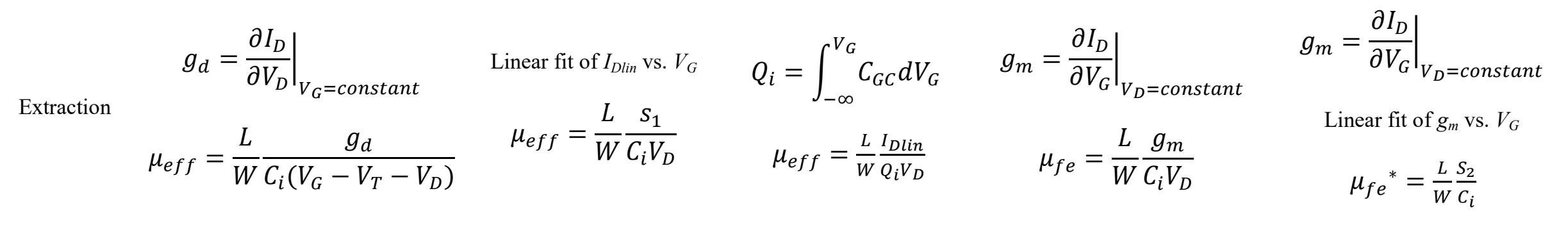

\begin{tabular}{|c|c|c|c|c|c|}
\hline Advantage & $\begin{array}{l}\text { Less contact effect, with } \\
V_{D} \text { and } V_{G} \text { dependences }\end{array}$ & Easy to use & $\begin{array}{l}\text { Accurate, no need of } C_{i} \\
\text { and } V_{T} \text {, reliable } V_{G} \text { de- } \\
\text { pendence }\end{array}$ & Easy to use & Relatively less contact effect \\
\hline $\begin{array}{l}\text { Disad- } \\
\text { vantage }\end{array}$ & Few data points for $V_{G}$ & $\begin{array}{l}\text { Sensitive to contact ef- } \\
\text { fect }\end{array}$ & $\begin{array}{l}\text { Additional } C-V \text { meas- } \\
\text { urements }\end{array}$ & $\begin{array}{c}\text { Sensitive to contact effect and } \\
\text { device operation }\end{array}$ & Rarely use \\
\hline
\end{tabular}




\section{WILEY-VCH}

Table 1 (continued): Summary of mobility extraction methods.

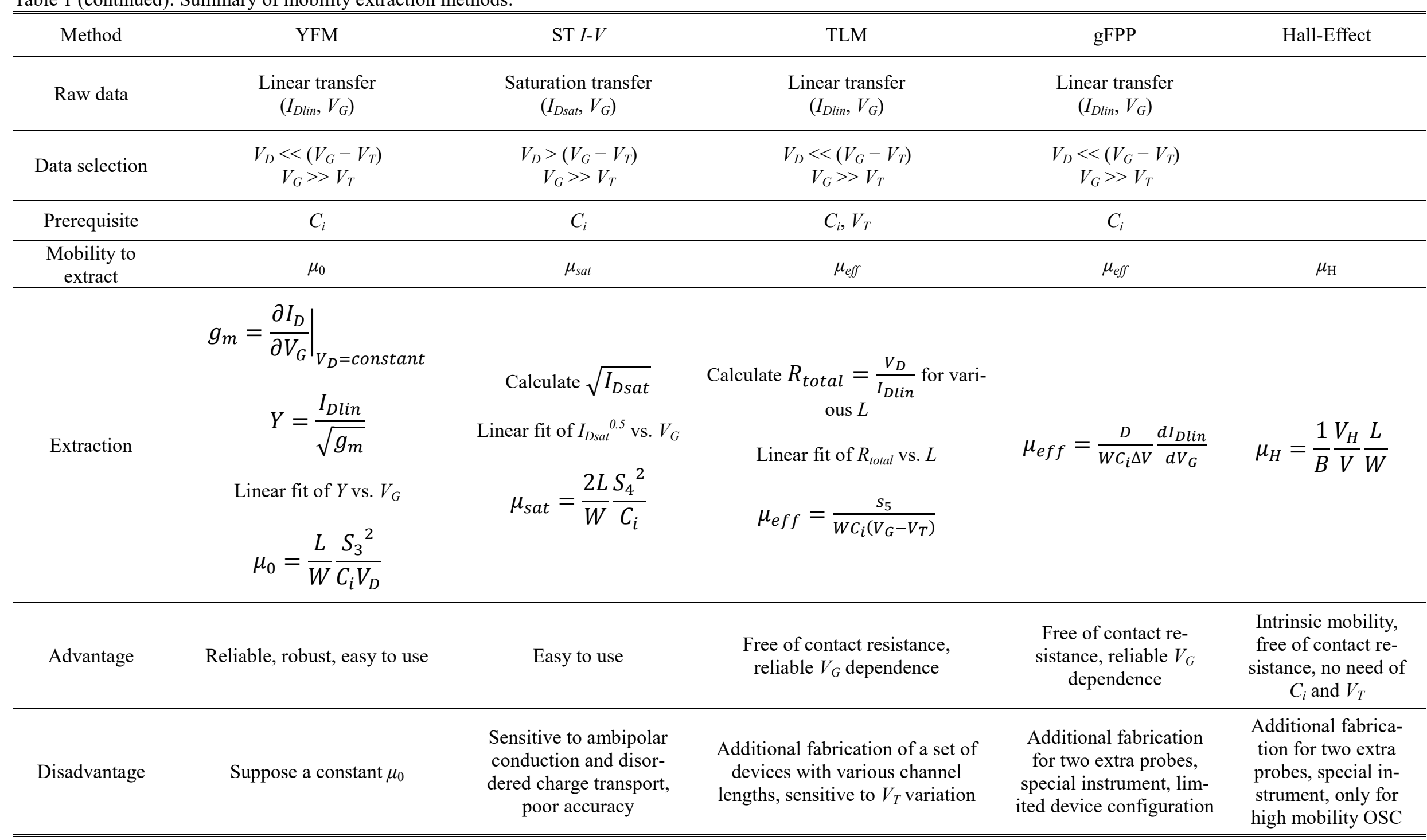




\section{WILEY-VCH}

[1] A. F. Paterson, S. Singh, K. J. Fallon, T. Hodsden, Y. Han, B. C. Schroeder, H. Bronstein, M. Heeney, I. McCulloch, T. D. Anthopoulos, Adv. Mater. 2018, 30.

[2] H. Sirringhaus, Adv. Mater. 2014, 26, 1319.

[3] I. McCulloch, A. Salleo, M. Chabinyc, Science 2016, 352, 1521.

[4] H. H. Choi, K. Cho, C. D. Frisbie, H. Sirringhaus, V. Podzorov, Nat. Mater. 2018, 17, 2.

[5] T. Uemura, C. Rolin, T.-H. Ke, P. Fesenko, J. Genoe, P. Heremans, J. Takeya, Adv. Mater. 2016, 28, 151.

[6] E. G. Bittle, J. I. Basham, T. N. Jackson, O. D. Jurchescu, D. J. Gundlach, Nat. Commun. 2016, 7, 10908.

[7] D. Venkateshvaran, M. Nikolka, A. Sadhanala, V. Lemaur, M. Zelazny, M. Kepa, M. Hurhangee, A. J. Kronemeijer, V. Pecunia, I. Nasrallah, I. Romanov, K. Broch, I. McCulloch, D. Emin, Y. Olivier, J. Cornil, D. Beljonne, H. Sirringhaus, Nature 2014, 515, 384.

[8] T. Okachi, Org. Electron. 2018, 57, 34.

[9] H. Y. Li, N. Tessler, J. L. Bredas, Adv. Funct. Mater. 2018, 28.

[10] C. Liu, G. T. Li, R. Di Pietro, J. Huang, Y. Y. Noh, X. Y. Liu, T. Minari, Phys. Rev. Applied 2017, 8, 034020.

[11] C. Liu, Y. Xu, Y.-Y. Noh, Mater. Today 2015, 18, 79.

[12] Y. Y. Hu, G. D. Li, W. Peng, Z. J. Chen, IEEE Electron Device Lett. 2018, 39, 421.

[13] Y. Xu, H. Sun, A. Liu, H. Zhu, B. Li, T. Minari, F. Balestra, G. Ghibaudo, Y.-Y. Noh, Adv. Funct. Mater. 2018, 28.

[14] Y. Xu, H. Sun, E.-Y. Shin, Y.-F. Lin, W. Li, Y.-Y. Noh, Adv. Mater. 2016, 28, 8531.

[15] Y. Xu, H. B. Sun, W. W. Li, Y. F. Lin, F. Balestra, G. Ghibaudo, Y. Y. Noh, Adv. Mater. 2017, 29, 1702729.

[16] J. Zaumseil, H. Sirringhaus, Chem. Rev. 2007, 107, 1296.

[17] S. M. Sze, Physics of Semiconductor Devices, JOHN WILEY\&SONS, Inc., NEW YORK 1981.

[18] T.-Y. Chu, O.-K. Song, Appl. Phys. Lett. 2007, 90, 203512.

[19] O. D. Jurchescu, M. Popinciuc, B. J. Van Wees, T. T. Palstra, Adv. Mater. 2007, 19, 688.

[20] J. Dacuna, A. Salleo, Phys. Rev. B 2011, 84.

[21] I. Campbell, D. Smith, C. Neef, J. Ferraris, Appl. Phys. Lett. 1999, 74, 2809.

[22] W.-Y. Hung, T.-H. Ke, Y.-T. Lin, C.-C. Wu, T.-H. Hung, T.-C. Chao, K.-T. Wong, C.-I. Wu, Appl. Phys. Lett. 2006, 88, 064102.

[23] S. Naka, H. Okada, H. Onnagawa, T. Tsutsui, Appl. Phys. Lett. 2000, 76, 197.

[24] R. Dost, A. Das, M. Grell, J. Appl. Phys. 2008, 104, 084519.

[25] A. Pivrikas, N. S. Sariciftci, G. Juška, R. Österbacka, Prog. Photovoltaics 2007, 15,

677.

[26] D. K. Schroder, Semiconductor material and device characterization, 3rd Edition, John wiley \& Sons, Inc., Hoboken, New Jersey 2006.

[27] Y. Xu, T. Minari, K. Tsukagoshi, J. A. Chroboczek, G. Ghibaudo, J. Appl. Phys. 2010, $107,114507$.

[28] G. Horowitz, R. Hajlaoui, H. Bouchriha, R. Bourguiga, M. Hajlaoui, Adv. Mater. 1998, $10,923$.

[29] S. G. J. Mathijssen, M.-J. Spijkman, A.-M. Andringa, P. A. van Hal, I. McCulloch, M. Kemerink, R. A. J. Janssen, D. M. de Leeuw, Adv. Mater. 2010, 22, 5105.

[30] Y. Xu, C. Liu, W. Scheideler, S. L. Li, W. W. Li, Y. F. Lin, F. Balestra, G. Ghibaudo, K. Tsukagoshi, IEEE Electron Device Lett. 2013, 34, 1298.

[31] K. Ryu, I. Kymissis, V. Bulovic, C. Sodini, IEEE Electron Device Lett. 2005, 26, 716. 


\section{WILEY-VCH}

[32] A. Wang, I. Kymissis, V. Bulovic, A. I. Akinwande, Appl. Phys. Lett. 2006, 89, 112109.

[33] G. Ghibaudo, Electron. Lett. 1988, 24, 543.

[34] G. Horowitz, R. Hajlaoui, D. Fichou, A. El Kassmi, J. Appl. Phys. 1999, 85, 3202.

[35] M. C. J. M. Vissenberg, M. Matters, Phys. Rev. B 1998, 57, 12964.

[36] D. W. He, J. S. Qiao, L. L. Zhang, J. Y. Wang, T. Lan, J. Qian, Y. Li, Y. Shi, Y. Chai, W. Lan, L. K. Ono, Y. B. Qi, J. B. Xu, W. Ji, X. R. Wang, Sci. Adv. 2017, 3.

[37] V. Podzorov, E. Menard, J. A. Rogers, M. E. Gershenson, Phys. Rev. Lett. 2005, 95, 226601.

[38] V. Podzorov, E. Menard, A. Borissov, V. Kiryukhin, J. A. Rogers, M. E. Gershenson, Phys. Rev. Lett. 2004, 93, 8.

[39] J. Takeya, J. Kato, K. Hara, M. Yamagishi, R. Hirahara, K. Yamada, Y. Nakazawa, S. Ikehata, K. Tsukagoshi, Y. Aoyagi, T. Takenobu, Y. Iwasa, Phys. Rev. Lett. 2007, 98, 196804.

[40] T. Sekitani, Y. Takamatsu, S. Nakano, T. Sakurai, T. Someya, Appl. Phys. Lett. 2006, 88.

[41] M. E. Gershenson, V. Podzorov, A. F. Morpurgo, Rev. Mod. Phys. 2006, 78, 973.

[42] Y. Xu, M. Benwadih, R. Gwoziecki, R. Coppard, T. Minari, C. Liu, K. Tsukagoshi, J. A. Chroboczek, F. Balestra, G. Ghibaudo, J. Appl. Phys. 2011, 110, 104513.

[43] C. Rolin, E. Kang, J. H. Lee, G. Borghs, P. Heremans, J. Genoe, Nat. Commun. 2017, 8.

[44] S. W. Luan, G. W. Neudeck, J. Appl. Phys. 1992, 72, 766.

[45] T. Minari, P. Darmawan, C. Liu, Y. Li, Y. Xu, K. Tsukagoshi, Appl. Phys. Lett. 2012, $100,093303$.

[46] Y. Xu, C. Liu, P. S. K. Amegadze, W. T. Park, D. X. Long, T. Minari, F. Balestra, G. Ghibaudo, Y. Y. Noh, Appl. Phys. Lett. 2014, 105, 133505.

[47] R. W. I. de Boer, N. N. Iosad, A. F. Stassen, T. M. Klapwijk, A. F. Morpurgo, Appl. Phys. Lett. 2005, 86.

[48] Y. Xu, C. Liu, D. Khim, Y.-Y. Noh, Phys. Chem. Chem. Phys. 2015, 17, 26553.

[49] Y. Xu, W. Scheideler, C. Liu, F. Balestra, G. Ghibaudo, K. Tsukagoshi, IEEE Electron Device Lett. 2013, 34, 535.

[50] J. Z. Wang, Z. H. Zheng, H. Sirringhaus, Appl. Phys. Lett. 2006, 89, 083513.

[51] K. Tsukagoshi, F. Fujimori, T. Minari, T. Miyadera, T. Hamano, Y. Aoyagi, Appl. Phys. Lett. 2007, 91, 113508.

[52] Y. Xu, H. Sun, A. Liu, H.-H. Zhu, W. Li, Y.-F. Lin, Y.-Y. Noh, Adv. Mater. 2018, 30, e1801830.

[53] R. Noriega, J. Rivnay, K. Vandewal, F. P. Koch, N. Stingelin, P. Smith, M. F. Toney, A. Salleo, Nat. Mater. 2013, 12, 1038.

[54] A. F. Paterson, N. D. Treat, W. M. Zhang, Z. P. Fei, G. Wyatt-Moon, H. Faber, G. Vourlias, P. A. Patsalas, O. Solomeshch, N. Tessler, M. Heeney, T. D. Anthopoulos, Adv. Mater. 2016, 28, 7791.

[55] D. Y. Khim, H. Han, K. J. Baeg, J. W. Kim, S. W. Kwak, D. Y. Kim, Y. Y. Noh, $A d v$. Mater. 2013, 25, 4302.

[56] S. G. Bucella, A. Luzio, E. Gann, L. Thomsen, C. R. McNeill, G. Pace, A. Perinot, Z. Chen, A. Facchetti, M. Caironi, Nat. Commun. 2015, 6, 8394.

[57] Y. B. Yuan, G. Giri, A. L. Ayzner, A. P. Zoombelt, S. C. B. Mannsfeld, J. H. Chen, D. Nordlund, M. F. Toney, J. S. Huang, Z. N. Bao, Nat. Commun. 2014, 5, 3005.

[58] Y. D. Park, J. A. Lim, H. S. Lee, K. Cho, Mater. Today 2007, 10, 46.

[59] L. L. Chua, J. Zaumseil, J. F. Chang, E. C. Ou, P. K. Ho, H. Sirringhaus, R. H. Friend, Nature 2005, 434, 194.

[60] A. F. Stassen, R. W. I. de Boer, N. N. Iosad, A. F. Morpurgo, Appl. Phys. Lett. 2004, 85,3899 . 


\section{WILEY-VCH}

[61] J. Veres, S. D. Ogier, S. W. Leeming, D. C. Cupertino, S. M. Khaffaf, Adv. Funct. Mater. 2003, 13, 199.

[62] N. G. Martinelli, M. Savini, L. Muccioli, Y. Olivier, F. Castet, C. Zannoni, D. Beljonne, J. Cornil, Adv. Funct. Mater. 2009, 19, 3254.

[63] S. J. Konezny, M. N. Bussac, L. Zuppiroli, Appl. Phys. Lett. 2009, 95, 263311.

[64] Y. Xu, H. Sun, Y. Y. Noh, IEEE Trans. Electron Dev. 2017, 64, 1932.

[65] D. Natali, M. Caironi, Adv. Mater. 2012, 24, 1357.

[66] D. Braga, G. Horowitz, Appl. Phs. A-Mater. 2009, 95, 193.

[67] Y. Xu, C. Liu, Y. Li, T. Minari, P. Darmawan, F. Balestra, G. Ghibaudo, K. Tsukagoshi, J. App. Phys. 2013, 113, 064507.

[68] Y. Xu, C. Liu, W. Scheideler, P. Darmawan, S. Li, F. Balestra, G. Ghibaudo, K. Tsukagoshi, Org. Electron. 2013, 14, 1797.

[69] D. Natali, J. R. Chen, F. Maddalena, F. G. Ferre, F. Di Fonzo, M. Caironi, $A d v$. Electron. Mater. 2016, 2, 1600097.

[70] F. Huang, A. Liu, H. Zhu, Y. Xu, F. Balestra, G. Ghibaudo, Y.-Y. Noh, J. Chu, W. Li, IEEE Electron Device Lett. 2019, 40, 605.

[71] S. Lee, A. Nathan, Science 2016, 354, 302.

[72] C. Jiang, H. W. Choi, X. Cheng, H. Ma, D. Hasko, A. Nathan, Science 2019, 363, 719.

[73] J. B. Kim, D. R. Lee, Appl. Phys. Lett. 2018, 112.

[74] Y. Xu, F. Balestra, G. Ghibaudo, Appl. Phys. Lett. 2011, 98, 233302.

[75] K. H. Liu, T. C. Chang, M. S. Wu, Y. S. Hung, P. H. Hung, T. Y. Hsieh, W. C. Chou, A. K. Chu, S. M. Sze, B. L. Yeh, Appl. Phys. Lett. 2014, 104.

[76] J. Lee, J. W. Chung, D. H. Kim, B. L. Lee, Park, J.I., S. Lee, R. Hausermann, B. Batlogg, S. S. Lee, I. Choi, I. W. Kim, M. S. Kang, J. Am. Chem. Soc. 2015, 137, 7990.

[77] T. Sakanoue, H. Sirringhaus, Nat. Mater. 2010, 9, 736.

[78] J. A. Letizia, J. Rivnay, A. Facchetti, M. A. Ratner, T. J. Marks, Adv. Funct. Mater. 2010, 20, 50 .

[79] N. I. Craciun, J. Wildeman, P. W. M. Blom, Phys. Rev. Lett. 2008, 100, 056601.

[80] D. Khim, Y. Xu, K. J. Baeg, M. Kang, W. T. Park, S. H. Lee, I. B. Kim, J. Kim, D. Y. Kim, C. Liu, Y. Y. Noh, Adv. Mater. 2016, 28, 518.

[81] S. Fratini, S. Ciuchi, Phys. Rev. Lett. 2009, 103, 266601.

[82] N. A. Minder, S. Ono, Z. H. Chen, A. Facchetti, A. F. Morpurgo, Adv. Mater. 2012, 24, 503.

[83] Y. Y. Hu, G. D. Li, Z. J. Chen, IEEE Electron Device Lett. 2018, 39, 276. 